\title{
Phthalocyanine-based Discotic Liquid Crystal Switching from Molten Alkyl Chain Type to Flying-seed-like Type
}

\author{
Hiromu Nakamura, ${ }^{a}$ Kouki Sugiyama, ${ }^{a}$ Kazuchika Ohta ${ }^{a}$ and Mikio Yasutake ${ }^{b}$ \\ Received (in $X X X, X X X)$ Xth $X X X X X X X X X 20 X X$, Accepted Xth $X X X X X X X X X 20 X X$ \\ ${ }_{5}$ DOI: 10.1039/b000000x
}

We have synthesised a series of the phthalocyanine-based discotic liquid crystals, $\left(m-\mathrm{C}_{n} \mathrm{OPhO}\right)_{8} \mathrm{PcCu} \quad(n$ $=1-20: \mathbf{2 a - 0})$, and investigated their mesomorphism by using a polarizing optical microscopy $(\mathrm{POM})$, a differential scanning calorimeter (DSC) and a temperature-dependent small angle X-ray diffractometer. We found that each of the derivatives 2a-o shows mesomorphism. However, the mesomorphism of the $10\left(m-\mathrm{C}_{n} \mathrm{OPhO}\right)_{8} \mathrm{PcCu}$ derivatives strongly depends on the alkoxy chain length $(n)$. The mesomorphism of the short chain-substituted derivatives $\mathbf{2 a - e}$ for $\mathrm{n}=1 \sim 5$ is flying-seed-like type induced by flip-flop of the peripheral bulky substituents, whereas the mesomorphism of the long chain-substituted derivatives $\mathbf{2 j - 0}$ for $\mathrm{n}=10 \sim 20$ is conventional molten alkyl chain type induced by melting of the long alkyl chains. The moderately long chain derivatives (2f-i) for $n=6-9$ in between show both types of mesophases. The

15 detailed temperature-dependent X-ray diffraction measurements were carried out for three representative derivatives, $\mathbf{2 b}(n=2$ for $n=1-5), \mathbf{2 h}(n=8$ for $n=6-9)$, and $\mathbf{2 o}(n=20$ for $n=10-20)$. As a result, we revealed that the $\mathrm{Col}_{\mathrm{ro}}(\mathrm{P} 2 \mathrm{~m})$ mesophase in $\mathbf{2 b}(n=2)$ gave a halo denoted as Halo arom. at $\mathrm{d} \cong 5.2 \AA$ due to flip-flop of the bulky aromatic substituents, and that the $\mathrm{Col}_{\text {ho }}$ mesophase in $\mathbf{2 0}(\mathrm{n}=20)$ gave a halo denoted as Halo $_{\text {alkyl }}$ at $\mathrm{d} \cong 4.6-4.8 \AA$ due to melting of the long alkyl chains. Therefore, we can 20 distinguish the type of mesophase from $\mathbf{H a l o}_{\text {arom. }}$ and $\mathbf{H a l o}_{\text {alkyl. }}$. Very interestingly, the $\left(m-\mathrm{C}_{8} \mathrm{OPhO}\right)_{8} \mathrm{PcCu}(\mathbf{2 h})$ derivative having moderately long alkyl chains gave $\mathbf{H a l o}_{\text {alkyl }}$ at about $4.8 \AA$ in the lower temperature mesophase of $\mathrm{Col}_{\mathrm{h}}$, but $\mathbf{H a l o}_{\text {arom }}$ at about $5.2 \AA$ in the higher temperature mesophase of $\mathrm{Col}_{\mathrm{ro}}\left(\mathrm{P} 2_{1} / \mathrm{a}\right)$ phase. This means that melting of the alkyl chains induces the $\mathrm{Col}_{\text {ho }}$ phase in the lower temperature region, but that flip-flop of the bulky aromatic substituents induces the $\mathrm{Col}_{\mathrm{ro}}\left(\mathrm{P}_{1} / \mathrm{a}\right)$ ${ }_{25}$ phase in the higher temperature region. This unusual reverse phase transition sequence from a higher symmetry of $\mathrm{Col}_{\mathrm{h}}$ mesophase to a lower symmetry of $\mathrm{Col}_{\mathrm{r}}$ mesophase on heating stage is attributable to such a unique stepwise melting of these two different types of substituents. To our best knowledge, this mesogen (2h) is the first example switching mesomorphism from molten alkyl chain type to flying-seed-like type in a discotic liquid crystal.

\section{${ }_{30}$ 1. INTRODUCTION}

Since the first liquid crystalline compounds were discovered by Reinitzer ${ }^{1}$ in 1888 , over 100,000 kinds of liquid crystalline compounds $^{2}$ have been synthesised up to date. These liquid crystalline compounds are generally classified into rod-like 35 molecules and discotic molecules judging from their molecular shapes, but both of them commonly have a structure having a rigid core at the centre and several flexible long alkyl chains in the periphery. When these liquid crystalline compounds are heated, the long alkyl chains melt at first to form a soft part, 40 whereas the central cores remain as a rigid part without melting, so that mesomorphism can be induced. Therefore, until now, we have long believed that it is essential for a liquid crystalline molecule to have a rigid core in the centre and flexible long alkyl chains in the periphery. However, it has been reported that a very ${ }_{45}$ few liquid crystalline compounds have neither a rigid core in the centre nor flexible long alkyl chains in the periphery. ${ }^{3-11}$

In 1911, Vorländer reported that sodium diphenylacetate, potassium dimethylacetate and potassium diethylacetate show mesomorphism although there is neither a rigid core in the centre ${ }_{50}$ nor flexible long alkyl chains in the periphery. ${ }^{3}$ However, he only reported that they show mesophases, but that neither their mesophase identification nor the mesomorphism induction mechanism was clarified. In the past 100 years, a very few researchers, Demus, ${ }^{4}$ Sanesi, ${ }^{5,}{ }^{6}$ Binnnemans, ${ }^{7}$ and our group, ${ }^{8}$ 55 have investigated these liquid crystalline compounds. In 2006, we clarified the mesomorphism induction mechanism of sodium diphenylacetate, potassium dimethylacetate and potassium diethylacetate by using temperature-dependent X-ray diffractometer. $^{8}$ 
Sodium diphenylacetate shows a hexagonal columnar $\left(\mathrm{Col}_{\mathrm{h}}\right)$ phase. On the other hand, potassium dimethylacetate and potassium diethylacetate show a smectic A (SmA) phase. These compounds form a soft part by free rotation of the bulky 5 substituents, diphenylmethyl, dimethylmethyl and diethylmethyl groups. Sodium metals form a one-dimensional nano-wire and diphenylmethyl groups freely rotate around the nano-wire to form an aromatic nanotube, so that sodium diphenylacetate shows a $\mathrm{Col}_{\mathrm{h}}$ phase. On the other hand, in potassium dimethylacetate and ${ }_{10}$ potassium diethylacetate, potassium metals form a two-dimensional potassium metal sheet and dimethylmethyl or diethylmethyl groups freely rotate to form an aliphatic sheet, so that they show a SmA phase. Such unique mesomorphism induction mechanism has never been reported so far. Since the 15 freely rotating substituents very resemble flying seeds of a maple tree, we named such type of liquid crystals as flying-seed-like liquid crystals. ${ }^{8}$

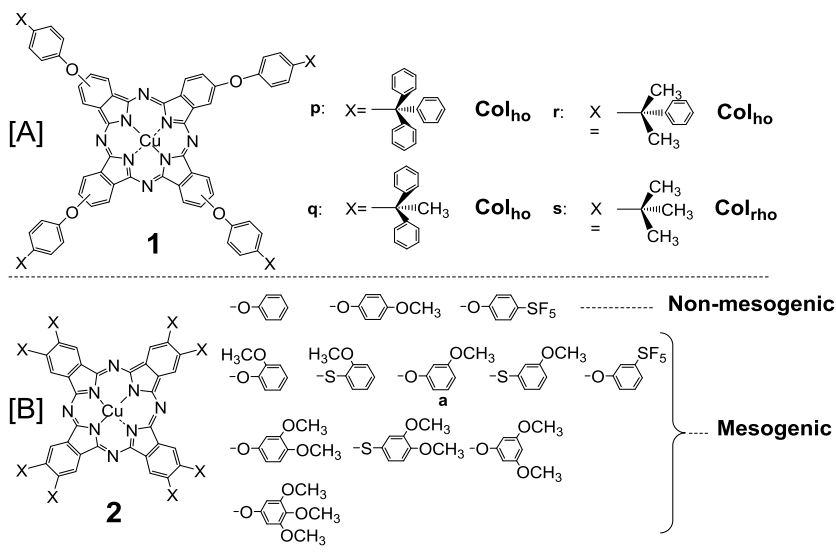

20 Fig. 1 Flying-seed-like liquid crystals based on phthalocyanine: [A] the first flying-seed-like liquid crystals, $1 \mathbf{p} \sim \mathbf{s}$, based on phthalocyanine. ${ }^{12-14}$; [B] dependence of liquid crystallinity on the substitution position for phthalocyanine-based compounds, 2. ${ }^{16-18}$

25 In 2009, Usol'tseva et al., reported a phthalocyanine derivative (1p in Figure 1A) substituted by four $p$-(triphenylmethyl)phenoxy groups shows a $\mathrm{Col}_{\mathrm{h}}$ phase, although it has no long alkyl chains in the periphery. ${ }^{12,13}$ In their reports, the mesophase was identified only by polarizing microscopic 30 observation (POM), without the X-ray diffraction measurement. In 2012, we therefore synthesised a series of phthalocyanine homologues (1p-s) shown in Figure $1 \mathrm{~A},{ }^{14}$ in order to investigate the detailed phase structures of these compounds by the temperature-dependent X-ray diffraction measurements. As a 35 result, we established that the homologue 1 p shows a $\mathrm{Col}_{\text {ho }}$ phase as reported by Usol'tseva et al. The phthalocyanine homologues 1q-r also show the same $\mathrm{Col}_{\text {ho }}$ phase, whereas the homologue $\mathbf{1 s}$ only shows a pseudohexagonal ordered columnar $\left(\mathrm{Col}_{\text {rho }}\right)$ phase. ${ }^{14}$ Generally, liquid crystalline phases give a halo observed at $c a .4 .5$ $40 \AA$ due to melting of the long alkyl chains. However, the present phthalocyanine homologues 1p-s gave an alternative halo at 5.7 $\AA \sim 6.9 \AA$ due to free rotation of the bulky substituents. It was confirmed from these phthalocyanine homologues (1p-s) that introduction of bulky substituents instead of long alkyl chains ${ }_{45}$ into the periphery of a rigid core can also induce mesomorphism.
In the past five years, we have explored several novel flying-seed-like liquid crystals. $^{14-19}$ Figure 1B shows our developed flying-seed-like phthalocyanine derivatives substituted by bulky substituents to induce mesomorphism. As can be seen 50 from this figure, the non-substituted phenoxy group, the $p$-methoxyphenoxy group and p-pentafluorosulfanylphenoxy group cannot induce mesomorphism. ${ }^{17,18}$ On the other hand, the compounds having a methoxy group at the ortho position or the meta position show mesomorphism. ${ }^{17}$ Interestingly, when a 55 methoxy group is introduced at least at the meta position in the phenoxy group, these compounds tend to show mesomorphism. ${ }^{17}$ When a phenoxy group is replaced by a phenylthio group, the compounds substituted by a methoxy group at the ortho and meta positions in the phenylthio also show mesomorphism. ${ }^{16}$ This 60 tendency could be seen also for the pentafluorosulfanyl substituted homologue. ${ }^{18}$ Thus, the introduction of the substituent at the $m$-position can induce the flying-seed-like mesomorphism. This means that rotation or flip-flop of the $m$-methoxyphenoxy groups may originate the large exclusion volume to form a soft ${ }_{65}$ part necessary to induce mesomorphism. ${ }^{17}$
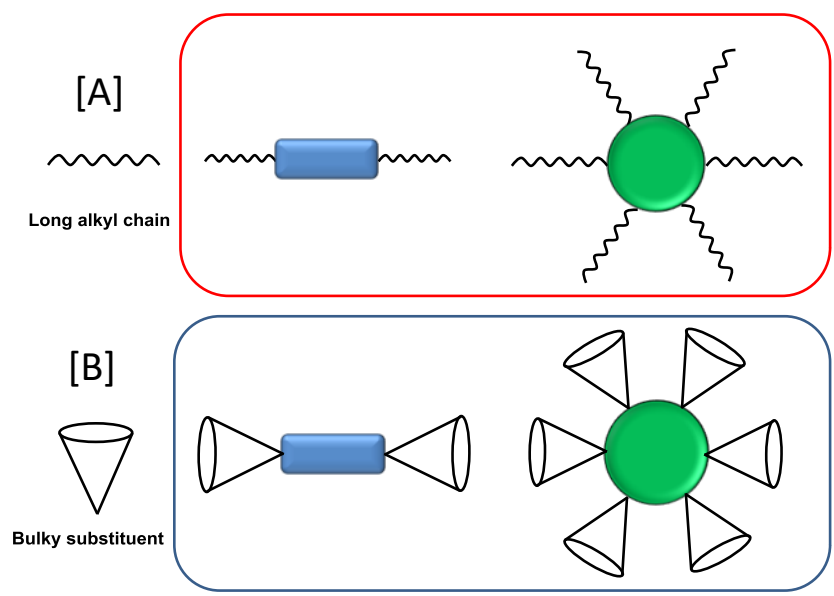

Fig. 2 Variety of liquid crystalline compounds: [A] conventional molten alkyl chain type of liquid crystals originated from melting of the long alkyl chains in the periphery, and [B] 70 flying-seed-like liquid crystals originated from free rotation of the bulky substituents in the periphery.

Therefore, mesomorphism can be induced from two different types, as illustrated in Figure 2: [A] melting of the long alkyl chains in the periphery to induce conventional molten alkyl 75 chain type of liquid crystals, and [B] free rotation or flip-flop of the bulky substituents in the periphery to induce novel flying-seed-like liquid crystals.

Hereupon, we focused on the phthalocyanine derivative $\left(m-\mathrm{C}_{1} \mathrm{OPhO}\right)_{8} \mathrm{PcCu} \quad(\mathbf{2 a}$ in Figure 1) showing columnar 80 mesomorphism. In this derivative (2a in Figure $3 \mathrm{~A}$ ), a very short methoxy group is substituted at the meta position of the phenoxy group. On the other hand, the homologous phthalocyanine derivative substituted by a long eicosanyloxy group at the meta position of the phenoxy group $\left(m-\mathrm{C}_{n} \mathrm{OPhO}\right)_{8} \mathrm{PcCu}(n=20: 20$ in ${ }_{85}$ Figure $3 \mathrm{~B}$ ) also show columnar mesomorphism, as previously reported. $^{20,} 21$ The flying-seed-like mesomorphism of the $\left(m-\mathrm{C}_{1} \mathrm{OPhO}\right)_{8} \mathrm{PcCu}(\mathbf{2 a})$ derivative may be induced by flip-flop of the peripheral bulky substituents, as shown in Figure 3A, whereas the mesomorphism of the $\left(m-\mathrm{C}_{20} \mathrm{OPhO}\right)_{8} \mathrm{PcCu}$ (2o) derivative 
may be induced by melting of the long alkyl chains like conventional liquid crystals, as shown in Figure 3B. Hence, mesomorphism of these two derivatives, $\mathbf{2 a}$ and $\mathbf{2 0}$, may be induced from different driving forces. If we will synthesise a 5 series of the $\left(m-\mathrm{C}_{n} \mathrm{OPhO}\right)_{8} \mathrm{PcCu}$ derivatives for $n=1-20$ to investigate their mesomorphism, we will be able to reveal the boundary of mesomorphism inducing driving forces switching from flying-seed-like type to molten alkyl chain type in the alkyl chain length, $n=1 \sim 20$.

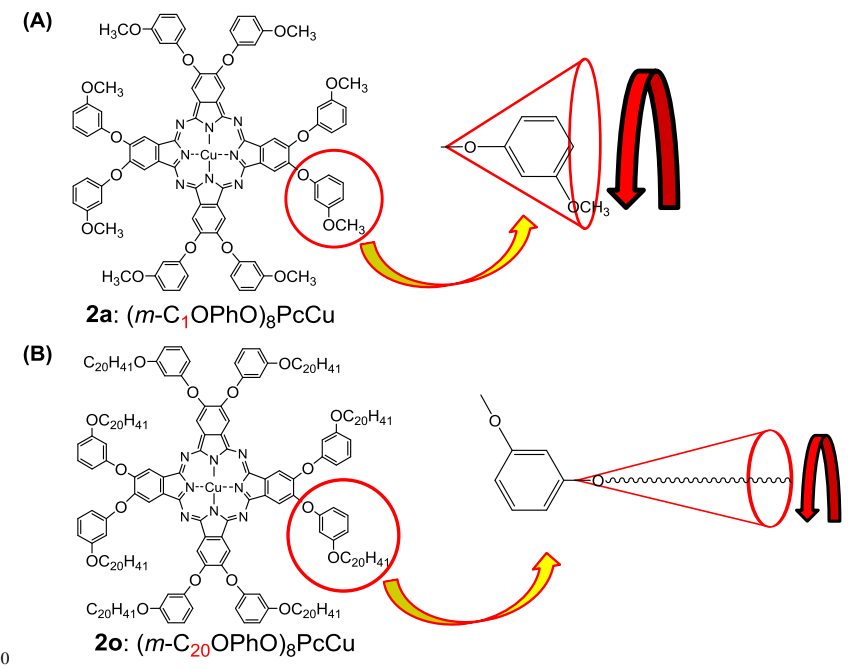

Fig. 3 Molecular formulae of liquid crystals based on phthalocyanine in our previous works: (A) flying-seed-like type liquid crystal, 2a, and (B) molten alkyl chain type liquid crystal, 20.

15 In this study, we have therefore synthesised additional homologues of $\left(m-\mathrm{C}_{n} \mathrm{OPhO}\right)_{8} \mathrm{PcCu}(n=2-9$ : $\mathbf{2 b}-\mathbf{i}$ in Scheme 1$)$ to investigate the boundary of the alkyl chain length in a series of the homologues 2a-o $(n=1 \sim 20)$. To our best knowledge, such a unique research has never been done up to date.

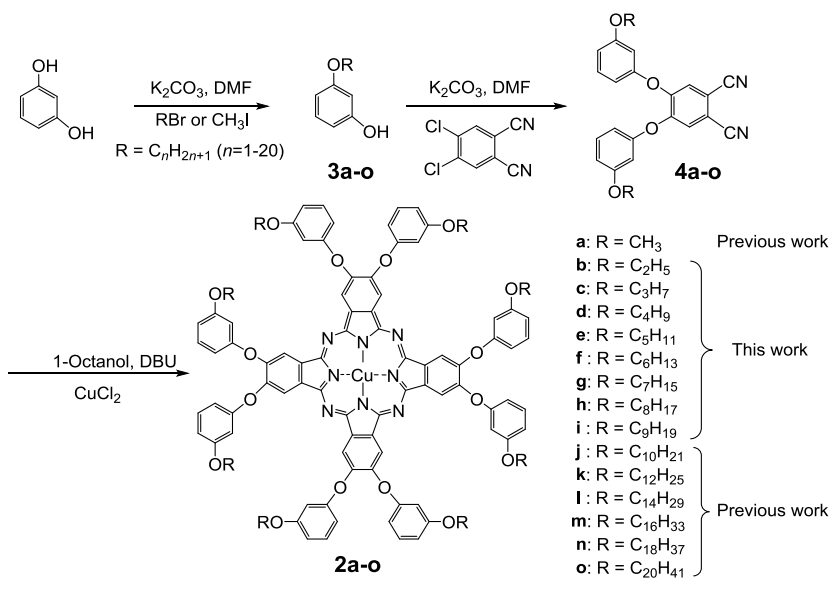

Scheme 1 Synthetic route for $\left(m-\mathrm{C}_{n} \mathrm{OPhO}\right)_{8} \mathrm{PcCu}(\mathrm{n}=1-20: \mathbf{2 a - o})$, $\mathrm{DMF}=\mathrm{N}, \mathrm{N}$-dimethylformamide and $\mathrm{DBU}=$

1,8-diazabicyclo[5,4,0]-undec-7-ene. $\mathbf{a}=$ Ref. $17, \mathbf{k}, \mathbf{l}=$ Ref. 20 and j, $\mathbf{m}^{\circ} \mathrm{o}=$ Ref. 21

\section{${ }_{25}$ 2. EXPERIMENTAL}

\section{2-1. Synthesis}

Scheme 1 shows synthetic route for octakis $(m-n$-alkoxyphenoxy)phthalocyaninato copper(II) $\left(m-\mathrm{C}_{n} \mathrm{OPhO}\right)_{8} \mathrm{PcCu}(n=2-9: \mathbf{2 b}-\mathbf{i})$. The synthesis was adopted our 30 previously reported methods. ${ }^{20,21}$ Phenol derivatives (3b-i) were synthesised by Williamson ether synthesis with resorcinol and the corresponding 1-bromoalkane purchased from Wako Pure Chemical Industries, Ltd. Phthalonitrile derivatives (4b-i) were synthesised by aromatic substitution with phenol derivatives 35 (3b-i) and 4,5-dichlorophthalonitrile purchased from Tokyo Chemical Industry (Tokyo Kasei). The target phthalocyanine derivatives (2b-i) were synthesised by cyclic tetramerization of the corresponding dicyano compounds (3b-i).

The detailed procedures are described below only for a 40 representative compound, $\left(m-\mathrm{C}_{2} \mathrm{OPhO}\right)_{8} \mathrm{PcCu}(\mathbf{2 b})$.

\section{3-Ethoxyphenol (3b)}

A mixture of resorcinol $(4.00 \mathrm{~g}, 36.4 \mathrm{mmol}), \mathrm{K}_{2} \mathrm{CO}_{3}(1.49 \mathrm{~g}, 10.8$ mmol) and dry DMF $(12.5 \mathrm{~mL})$ was stirred at $70^{\circ} \mathrm{C}$ under the nitrogen atmosphere for $25 \mathrm{~min}$. Then, bromoethane $(5.13 \mathrm{~g}, 47.1$

$45 \mathrm{mmol}$ ) was added to the mixture and it was stirred at $95^{\circ} \mathrm{C}$ under the nitrogen atmosphere for $3 \mathrm{~h} 50 \mathrm{~min}$. The reaction mixture was extracted with chloroform and washed with water. The organic layer was dried over $\mathrm{Na}_{2} \mathrm{SO}_{4}$ for $1 \mathrm{~h}$. After removing the $\mathrm{Na}_{2} \mathrm{SO}_{4}$ by filtration, the filtrate was evaporated in vacuum using an 50 evaporator. The residue was purified by column chromatography (silica gel, chloroform, $R_{\mathrm{f}}=0.25$ ) to obtain $0.276 \mathrm{~g}$ of reddish brown liquid. Yield $=55.3 \%$. ${ }^{1} \mathrm{H}$ NMR $\left(400 \mathrm{MH}_{\mathrm{z}} ; \mathrm{CDCl}_{3}\right.$; $\left.\mathrm{Me}_{4} \mathrm{Si}\right): \delta(\mathrm{ppm})=1.39\left(3 \mathrm{H}, \mathrm{t}, J=7.2 \mathrm{H}_{\mathrm{z}},-\mathrm{CH}_{3}\right), 4.00(2 \mathrm{H}, \mathrm{q}, J$ $\left.=6.9 \mathrm{H}_{\mathrm{z}},-\mathrm{OCH}_{2^{-}}\right), 5.72(1 \mathrm{H}, \mathrm{s}, \mathrm{Ar}-\mathrm{OH}), 6.41-6.44(2 \mathrm{H}, \mathrm{m}$, $\left.{ }_{55} \mathrm{Ar}-\mathrm{H}\right), 6.45-6.48(1 \mathrm{H}, \mathrm{m}, \mathrm{Ar}-\mathrm{H})$ and $7.10\left(1 \mathrm{H}, \mathrm{t}, J=8.4 \mathrm{H}_{\mathrm{z}}\right.$, Ar-H).

Since other homologues $(\mathbf{3 c}-\mathbf{i})$ were synthesised in the same way, only the yields and ${ }^{1} \mathrm{H}-\mathrm{NMR}$ data are described below.

3-Propoxyphenol (3c)

${ }_{60}$ Yield $=34.5 \%$. Liquid at rt. ${ }^{1} \mathrm{H}$ NMR (400 $\mathrm{MH}_{\mathrm{Z}}$; DMSO; $\left.\mathrm{Me}_{4} \mathrm{Si}\right): \delta(\mathrm{ppm})=0.96\left(3 \mathrm{H}, \mathrm{t}, J=7.4 \mathrm{H}_{\mathrm{Z}},-\mathrm{CH}_{3}\right), 1.70(2 \mathrm{H}$, sext., $\left.J=7.0 \mathrm{H}_{\mathrm{Z}},-\mathrm{CH}_{2^{-}}\right), 3.84\left(2 \mathrm{H}, \mathrm{t}, J=6.6 \mathrm{H}_{\mathrm{Z}},-\mathrm{OCH}_{2^{-}}\right)$, 6.31-6.36 (3 H, m, Ar-H), $7.03\left(1 \mathrm{H}, \mathrm{t}, J=8.0 \mathrm{H}_{\mathrm{Z}}, \mathrm{Ar}-\mathrm{H}\right)$ and $9.35(1 \mathrm{H}, \mathrm{s}, \mathrm{Ar}-\mathrm{OH})$.

\section{3-Buthoxyphenol (3d)}

Yield $=35.7 \%$. Liquid at rt. ${ }^{1} \mathrm{H}$ NMR $\left(400 \mathrm{MH}_{\mathrm{Z}} ; \mathrm{CDCl}_{3} ; \mathrm{Me}_{4} \mathrm{Si}\right)$ : $\delta(\mathrm{ppm})=0.90\left(3 \mathrm{H}, \mathrm{t}, J=7.4 \mathrm{~Hz},-\mathrm{CH}_{3}\right), 1.41(2 \mathrm{H}$, sext., $J=7.6$ $\left.\mathrm{Hz},-\mathrm{CH}_{2^{-}}\right), 1.68\left(2 \mathrm{H}\right.$, quin., $\left.J=6.1 \mathrm{~Hz},-\mathrm{CH}_{2}-\right), 3.86(2 \mathrm{H}, \mathrm{t}, J=$ $\left.6.8 \mathrm{~Hz},-\mathrm{OCH}_{2}-\right), 4.99(1 \mathrm{H}, \mathrm{s}, \mathrm{Ar}-\mathrm{OH}), 6.32-6.35(2 \mathrm{H}, \mathrm{m}, \mathrm{Ar}-\mathrm{H})$, 70 6.40-6.42 (1 H, m, Ar-H) and $7.04(1 \mathrm{H}, \mathrm{t}, J=8.4 \mathrm{~Hz}, \mathrm{Ar}-\mathrm{H})$.

\section{3-Pentyloxyphenol (3e)}

Yield $=20.5 \%$. Liquid at rt. ${ }^{1} \mathrm{H}$ NMR $\left(400 \mathrm{MH}_{\mathrm{z}} ; \mathrm{CDCl}_{3} ; \mathrm{Me}_{4} \mathrm{Si}\right)$ : $\delta(\mathrm{ppm})=0.96\left(3 \mathrm{H}, \mathrm{t}, J=7.1 \mathrm{H}_{\mathrm{z}},-\mathrm{CH}_{3}\right), 1.36-1.49(4 \mathrm{H}, \mathrm{m}$, $\left.-\mathrm{C}_{2} \mathrm{H}_{4^{-}}\right), 1.80\left(2 \mathrm{H}\right.$, quin., $\left.J=7.0 \mathrm{H}_{\mathrm{z}},-\mathrm{CH}_{2^{-}}\right), 3.95(2 \mathrm{H}, \mathrm{t}, J=6.6$ $\left.{ }_{75} \mathrm{H}_{\mathrm{z}},-\mathrm{OCH}_{2^{-}}\right), 5.40(1 \mathrm{H}, \mathrm{s}, \mathrm{Ar}-\mathrm{OH}), 6.43-6.46(2 \mathrm{H}, \mathrm{m}, \mathrm{Ar}-\mathrm{H})$, 6.50-6.53 (1 H, m, Ar-H) and $7.14(1 \mathrm{H}, \mathrm{t}, J=8.6 \mathrm{~Hz}, \mathrm{Ar}-\mathrm{H})$

\section{3-Heptyloxyphenol (3g)}

Yield $=18.5 \%$. Liquid at rt. ${ }^{1} \mathrm{H}$ NMR $\left(400 \mathrm{MH}_{\mathrm{z}} ; \mathrm{CDCl}_{3} ; \mathrm{Me}_{4} \mathrm{Si}\right)$ : $\delta(\mathrm{ppm})=0.93\left(3 \mathrm{H}, \mathrm{t}, J=7.0 \mathrm{H}_{\mathrm{z}},-\mathrm{CH}_{3}\right), 1.30-1.50(8 \mathrm{H}, \mathrm{m}$, $\left.{ }_{80}-\mathrm{C}_{4} \mathrm{H}_{8^{-}}\right), 1.79\left(2 \mathrm{H}\right.$, quin., $\left.J=7.0 \mathrm{H}_{\mathrm{z}},-\mathrm{CH}_{2^{-}}\right), 3.94(2 \mathrm{H}, \mathrm{t}, J=6.6$ $\left.\mathrm{H}_{\mathrm{z}},-\mathrm{OCH}_{2}-\right), 5.34(1 \mathrm{H}, \mathrm{s}, \mathrm{Ar}-\mathrm{OH}), 6.43-6.46(2 \mathrm{H}, \mathrm{m}, \mathrm{Ar}-\mathrm{H})$, 6.50-6.53 (1 H, m, Ar-H) and $7.14(1 \mathrm{H}, \mathrm{t}, J=8.4 \mathrm{~Hz}, \mathrm{Ar}-\mathrm{H})$.

3-Octyloxyphenol (3h)

Yield $=48.3 \%$. Liquid at rt. ${ }^{1} \mathrm{H}$ NMR $\left(400 \mathrm{MH}_{\mathrm{z}} ; \mathrm{CDCl}_{3} ; \mathrm{Me}_{4} \mathrm{Si}\right)$ : ${ }_{85} \delta(\mathrm{ppm})=0.91\left(3 \mathrm{H}, \mathrm{t}, J=7.0 \mathrm{H}_{\mathrm{z}},-\mathrm{CH}_{3}\right), 1.31-1.50(8 \mathrm{H}, \mathrm{m}$, 
$\left.-\mathrm{C}_{5} \mathrm{H}_{10^{-}}\right), 1.79\left(2 \mathrm{H}\right.$, quin., $\left.J=7.0 \mathrm{H}_{\mathrm{z}},-\mathrm{CH}_{2^{-}}\right), 3.95(2 \mathrm{H}, \mathrm{t}, J=6.6$ $\left.\mathrm{H}_{\mathrm{z}},-\mathrm{OCH}_{2}-\right), 5.40(1 \mathrm{H}, \mathrm{s}, \mathrm{Ar}-\mathrm{OH}), 6.43-6.46(2 \mathrm{H}, \mathrm{m}, \mathrm{Ar}-\mathrm{H})$, 6.50-6.53 (1 H, m, Ar-H) and $7.14(1 \mathrm{H}, \mathrm{t}, J=8.4 \mathrm{~Hz}, \mathrm{Ar}-\mathrm{H})$.

3-Nonyloxyphenol (3i)

${ }_{5}$ Yield $=16.3 \%$. Liquid at rt. ${ }^{1} \mathrm{H} \mathrm{NMR}\left(400 \mathrm{MH}_{\mathrm{z}} ; \mathrm{CDCl}_{3} ; \mathrm{Me}_{4} \mathrm{Si}\right)$ : $\delta(\mathrm{ppm})=0.92\left(3 \mathrm{H}, \mathrm{t}, J=6.8 \mathrm{H}_{\mathrm{z}},-\mathrm{CH}_{3}\right), 1.27-1.50(12 \mathrm{H}, \mathrm{m}$, $\left.-\mathrm{C}_{6} \mathrm{H}_{12^{-}}\right), 1.79$ (2 H, quin., $\left.J=7.2 \mathrm{H}_{\mathrm{z}},-\mathrm{CH}_{2^{-}}\right), 3.94(2 \mathrm{H}, \mathrm{t}, J=6.6$ $\left.\mathrm{H}_{\mathrm{z}},-\mathrm{OCH}_{2}-\right), 5.07$ (1 H, s, Ar-OH), 6.42-6.45 (2 H, m, Ar-H), 6.50-6.53 (1 H, m, Ar-H) and $7.14(1 \mathrm{H}, \mathrm{t}, J=8.4 \mathrm{~Hz}, \mathrm{Ar}-\mathrm{H})$.

10 4,5-Bis(3-ethoxyphenoxy)phthalonitrile (4b)

A mixture of 3-ethoxyphenol (3b) $(0.624 \mathrm{~g}, 4.52 \mathrm{mmol}), \mathrm{K}_{2} \mathrm{CO}_{3}$ $(0.632 \mathrm{~g}, 4.57 \mathrm{mmol})$ and dry DMF $(30 \mathrm{~mL})$ was stirred at $90^{\circ} \mathrm{C}$ under the nitrogen atmosphere for $10 \mathrm{~min}$. Then, 4,5-dichlorophtalonitrile $(0.394 \mathrm{~g}, 2.00 \mathrm{mmol})$ was added to the 15 mixture and it was stirred at $100^{\circ} \mathrm{C}$ under the nitrogen atmosphere for $1 \mathrm{~h} 50 \mathrm{~min}$. The reaction mixture was extracted with chloroform and washed with water. The organic layer was dried over $\mathrm{Na}_{2} \mathrm{SO}_{4}$ for $1 \mathrm{~h}$. After removing the $\mathrm{Na}_{2} \mathrm{SO}_{4}$ by filtration, the filtrate was evaporated in vacuum using an evaporator. The 20 residue was purified by column chromatography (silica gel, toluene, $R_{\mathrm{f}}=0.25$ ) to obtain $0.423 \mathrm{~g}$ of light blue solid. Yield = $51.5 \%$. M.p. $=125.0 \sim 125.4^{\circ} \mathrm{C} .{ }^{1} \mathrm{H} \mathrm{NMR}\left(400 \mathrm{MH}_{\mathrm{z}} ; \mathrm{CDCl}_{3}\right.$; $\left.\mathrm{Me}_{4} \mathrm{Si}\right): \delta(\mathrm{ppm})=1.37\left(6 \mathrm{H}, \mathrm{t}, J=7.0 \mathrm{H}_{\mathrm{z}},-\mathrm{CH}_{3}\right), 3.78(4 \mathrm{H}, \mathrm{q}, J$ $\left.=6.9 \mathrm{H}_{\mathrm{z}},-\mathrm{OCH}_{2^{-}}\right), 6.53-6.58(4 \mathrm{H}, \mathrm{m}, \mathrm{Ar}-\mathrm{H}), 6.79-6.81(2 \mathrm{H}, \mathrm{m}$,

$\left.{ }_{25} \mathrm{Ar}-\mathrm{H}\right), 7.04(2 \mathrm{H}, \mathrm{s}, \mathrm{Ar}-\mathrm{H})$ and $7.30\left(2 \mathrm{H}, \mathrm{t}, J=8.2 \mathrm{H}_{\mathrm{z}}, \mathrm{Ar}-\mathrm{H}\right)$. IR: $\left(\mathrm{KBr}, v_{\max } / \mathrm{cm}^{-1}\right) 2241(-\mathrm{CN})$.

Since other homologues $(\mathbf{4 c - i})$ were synthesised in the same way, only the yields, ${ }^{1} \mathrm{H}-\mathrm{NMR}$ data and FT-IR data are described below.

${ }_{30}$ 4,5-Bis(3-propooxyphenoxy)phthalonitrile (4c)

Yield $=86.1 \%$. M.p. $=38.0 \sim 38.5^{\circ} \mathrm{C} .{ }^{1} \mathrm{H}$ NMR $\left(400 \mathrm{MH}_{\mathrm{Z}}\right.$; $\left.\mathrm{CDCl}_{3} ; \mathrm{Me}_{4} \mathrm{Si}\right): \delta(\mathrm{ppm})=1.07\left(6 \mathrm{H}, \mathrm{t}, J=7.2 \mathrm{~Hz},-\mathrm{CH}_{3}\right), 1.84(4$ $\mathrm{H}$, sext, $\left.J=7.0 \mathrm{~Hz},-\mathrm{CH}_{2}-\right), 3.95\left(4 \mathrm{H}, \mathrm{t}, J=6.6 \mathrm{~Hz},-\mathrm{OCH}_{2^{-}}\right)$, 6.63-6.67 (4 H, m, Ar-H), 6.83-6.86 (2 H, m, Ar-H), $7.22(1 \mathrm{H}, \mathrm{s}$, $35 \mathrm{Ar}-\mathrm{H})$ and $7.35(2 \mathrm{H}, \mathrm{t}, J=8.2 \mathrm{~Hz}, \mathrm{Ar}-\mathrm{H})$. IR: $\left(\mathrm{KBr}, v_{\max } / \mathrm{cm}^{-1}\right)$ $2238(-\mathrm{CN})$.

\section{4,5-Bis(3-buthoxyphenoxy)phthalonitrile (4d)}

Yield $=19.5 \%$. Liquid at rt. ${ }^{1} \mathrm{H}$ NMR $\left(400 \mathrm{MH}_{\mathrm{Z}} ; \mathrm{CDCl}_{3} ; \mathrm{Me}_{4} \mathrm{Si}\right)$ : $\delta(\mathrm{ppm})=1.00\left(6 \mathrm{H}, \mathrm{t}, J=7.4 \mathrm{~Hz},-\mathrm{CH}_{3}\right), 1.52(4 \mathrm{H}$, sext., $J=7.6$ $\left.{ }_{40} \mathrm{~Hz},-\mathrm{CH}_{2^{-}}\right), 1.80\left(4 \mathrm{H}\right.$, quin., $\left.J=6.0 \mathrm{~Hz},-\mathrm{CH}_{2}-\right), 3.98(4 \mathrm{H}, \mathrm{t}, J=$ $\left.6.4 \mathrm{~Hz},-\mathrm{OCH}_{2^{-}}\right), 6.63-6.67(4 \mathrm{H}, \mathrm{m}, \mathrm{Ar}-\mathrm{H}), 6.82-6.85(2 \mathrm{H}, \mathrm{m}$, Ar-H), 7.23 (1 H, s, Ar-H) and $7.34(2 \mathrm{H}, \mathrm{t}, J=8.2 \mathrm{~Hz}, \mathrm{Ar}-\mathrm{H})$. IR: $\left(\mathrm{KBr}, v_{\max } / \mathrm{cm}^{-1}\right) 2233(-\mathrm{CN})$.

4,5-Bis(3-pentyloxyphenoxy)phthalonitrile (4e)

${ }_{45}$ Yield $=73.4 \%$. Liquid at rt. ${ }^{1} \mathrm{H}$ NMR $\left(400 \mathrm{MH}_{\mathrm{z}} ; \mathrm{CDCl}_{3} ; \mathrm{Me}_{4} \mathrm{Si}\right)$ : $\delta(\mathrm{ppm})=0.86\left(6 \mathrm{H}, \mathrm{t}, J=7.0 \mathrm{H}_{\mathrm{z}},-\mathrm{CH}_{3}\right), 1.27-1.41(8 \mathrm{H}, \mathrm{m}$, $-\mathrm{C}_{2} \mathrm{H}_{4^{-}}$), $1.72\left(4 \mathrm{H}\right.$, quin., $\left.J=7.0 \mathrm{~Hz},-\mathrm{C}_{2} \mathrm{H}_{4^{-}}\right), 3.88(4 \mathrm{H}, \mathrm{t}, J=6.6$ $\left.\mathrm{H}_{\mathrm{z}},-\mathrm{OCH}_{2^{-}}\right), 6.53-6.57$ (4 H, m, Ar-H), 6.72-6.75 (2 H, m, Ar-H), 7.13(2 H, s, Ar-H) and $7.25(2 \mathrm{H}, \mathrm{t}, J=8.0 \mathrm{~Hz}, \mathrm{Ar}-\mathrm{H})$. IR: $(\mathrm{KBr}$, $\left.50 v_{\max } / \mathrm{cm}^{-1}\right) ; 2233(-\mathrm{CN})$.

\section{4,5-Bis(3-heptyloxyphenoxy)phthalonitrile (4g)}

Yield $=98.7 \%$. Liquid at rt. ${ }^{1} \mathrm{H}$ NMR $\left(400 \mathrm{MH}_{\mathrm{z}} ; \mathrm{CDCl}_{3} ; \mathrm{Me}_{4} \mathrm{Si}\right)$ : $\delta(\mathrm{ppm})=0.82\left(6 \mathrm{H}, \mathrm{t}, J=6.8 \mathrm{H}_{\mathrm{z}},-\mathrm{CH}_{3}\right), 1.19-1.42(16 \mathrm{H}, \mathrm{m}$, $-\mathrm{C}_{4} \mathrm{H}_{8^{-}}$), 1.72 (4 H, quin., $\left.J=7.0 \mathrm{~Hz},-\mathrm{CH}_{2^{-}}\right), 3.88(4 \mathrm{H}, \mathrm{t}, J=6.4$ $\left.{ }_{55} \mathrm{H}_{\mathrm{z}},-\mathrm{OCH}_{2}-\right)$, 6.53-6.57 (4 H, m, Ar-H), 6.72-6.75 (2 H, m, Ar-H), 7.13(2 H, s, Ar-H) and $7.25(2 \mathrm{H}, \mathrm{t}, J=8.4 \mathrm{~Hz}, \mathrm{Ar}-\mathrm{H})$. IR: $(\mathrm{KBr}$, $\left.v_{\max } / \mathrm{cm}^{-1}\right) 2233(-\mathrm{CN})$.

4,5-Bis(3-octyloxyphenoxy)phthalonitrile (4h)

Yield $=75.9 \%$. Liquid at rt. ${ }^{1} \mathrm{H}$ NMR $\left(400 \mathrm{MH}_{z} ; \mathrm{CDCl}_{3} ; \mathrm{Me}_{4} \mathrm{Si}\right)$ :
${ }_{60} \delta(\mathrm{ppm})=0.82\left(6 \mathrm{H}, \mathrm{t}, J=7.0 \mathrm{H}_{\mathrm{z}},-\mathrm{CH}_{3}\right), 1.21-1.42(16 \mathrm{H}, \mathrm{m}$, $\left.-\mathrm{C}_{5} \mathrm{H}_{10^{-}}\right), 1.72\left(4 \mathrm{H}\right.$, quin., $\left.J=7.0 \mathrm{~Hz},-\mathrm{CH}_{2^{-}}\right), 3.88(4 \mathrm{H}, \mathrm{t}, J=6.6$ $\left.\mathrm{H}_{\mathrm{z}},-\mathrm{OCH}_{2}-\right)$, 6.53-6.57 (4 H, m, Ar-H), 6.73-6.75 (2 H, m, Ar-H), $7.13(2 \mathrm{H}, \mathrm{s}, \mathrm{Ar}-\mathrm{H})$ and $7.25(2 \mathrm{H}, \mathrm{t}, J=8.4 \mathrm{~Hz}, \mathrm{Ar}-\mathrm{H})$. IR: $(\mathrm{KBr}$, $\left.v_{\max } / \mathrm{cm}^{-1}\right) 2233(-\mathrm{CN})$.

\section{4,5-Bis(3-nonyloxyphenoxy)phthalonitrile (4i)}

Yield $=75.9 \%$. M.p. $=38.0 \sim 38.5^{\circ} \mathrm{C} .{ }^{1} \mathrm{H} \mathrm{NMR}\left(400 \mathrm{MH}_{\mathrm{z}} ; \mathrm{CDCl}_{3}\right.$; $\left.\mathrm{Me}_{4} \mathrm{Si}\right): \delta(\mathrm{ppm})=0.81\left(6 \mathrm{H}, \mathrm{t}, J=6.8 \mathrm{H}_{\mathrm{z}},-\mathrm{CH}_{3}\right) 1.20-1.42(20 \mathrm{H}$, m, $\left.-\mathrm{C}_{6} \mathrm{H}_{12^{-}}\right), 1.71\left(4 \mathrm{H}\right.$, quin., $\left.J=7.0 \mathrm{~Hz},-\mathrm{C}_{2} \mathrm{H}_{4}-\right), 3.87(4 \mathrm{H}, \mathrm{t}, J$ $\left.=6.6 \mathrm{H}_{\mathrm{z}},-\mathrm{OCH}_{2^{-}}\right), 6.53-6.57(4 \mathrm{H}, \mathrm{m}, \mathrm{Ar}-\mathrm{H}), 6.72-6.75(2 \mathrm{H}, \mathrm{m}$, $70 \mathrm{Ar}-\mathrm{H}), 7.13(2 \mathrm{H}, \mathrm{s}, \mathrm{Ar}-\mathrm{H})$ and $7.25(2 \mathrm{H}, \mathrm{t}, J=8.0 \mathrm{~Hz}, \mathrm{Ar}-\mathrm{H})$. IR: $\left(\mathrm{KBr}, v_{\max } / \mathrm{cm}^{-1}\right) 2237(-\mathrm{CN})$.

\section{$\left(\mathrm{m}-\mathrm{C}_{2} \mathrm{OPhO}\right)_{8} \mathrm{PcCu}(2 \mathrm{~b})$}

A mixture of 4,5-bis(3-ethoxyphenoxy)phthalonitrile (4b) (0.201 g, $0.502 \mathrm{mmol})$, 1-octatanol $(5 \mathrm{~mL}), \mathrm{CuCl}_{2}(0.022 \mathrm{~g} 、 0.162$ $75 \mathrm{mmol}$ ) and DBU (4 drops) was refluxed under the nitrogen atmosphere for $3 \mathrm{~h} 10 \mathrm{~min}$. Methanol was poured into the reaction mixture to precipitate the target compound. The methanolic layer was removed by filtration and then resulting precipitate was washed with methanol, ethanol and acetone, 80 respectively. The residue was purified by hot filtration with chloroform to obtain $0.137 \mathrm{~g}$ of green solid. Yield $=65.3 \%$.

Yields, elemental analysis data and MALDI-TOF mass data: See Table 1.UV-vis spectral data: See Table 2.

The other homologues (2c-i) could be similarly synthesised. ${ }_{85}$ The yields, elemental analysis data and MALDI-TOF mass data are shown in Table 1 and the UV-Vis spectrum data are summarized in Table 2.

Table 1. TOF-Mass spectral data, elemental analysis data and yields of $90\left(m-\mathrm{C}_{\mathrm{n}} \mathrm{OPhO}\right)_{8} \mathrm{PcCu}(\mathrm{n}=\mathbf{2 - 9} \mathbf{2} \mathbf{2 b - i})$.

\begin{tabular}{|c|c|c|c|c|c|c|c|}
\hline \multirow{2}{*}{ Compound } & \multirow{2}{*}{$\begin{array}{l}\text { Mol. formula } \\
\text { (Mol. wt) }\end{array}$} & \multicolumn{2}{|c|}{ Exact mass } & \multicolumn{3}{|c|}{ Elemental analysis: Found(Calcd.)(\%) } & \multirow{2}{*}{ Yield(\% } \\
\hline & & Calcd. & Observed & c & $\mathrm{H}$ & $\mathrm{N}$ & \\
\hline 2b: $\left(m-\mathrm{C}_{2} \mathrm{OPhO}\right)_{8} \mathrm{PcCu}$ & $\begin{array}{c}\mathrm{C}_{96} \mathrm{H}_{80} \mathrm{CuN}_{8} \mathrm{O}_{16} \\
(1665.25)\end{array}$ & 1663.32 & 1663.32 & $\begin{array}{l}69.30 \\
(69.24)\end{array}$ & $\begin{array}{l}5.00 \\
(4.84)\end{array}$ & $\begin{array}{l}7.05 \\
(6.73)\end{array}$ & 65.3 \\
\hline $2 \mathrm{c}:\left(m-\mathrm{C}_{3} \mathrm{OPhO}\right)_{8} \mathrm{PcCu}$ & $\begin{array}{c}\mathrm{C}_{104} \mathrm{H}_{96} \mathrm{CuN}_{8} \mathrm{O}_{16} \\
(1777.47)\end{array}$ & 1775.62 & 1775.49 & $\begin{array}{c}70.44 \\
(70.27)\end{array}$ & $\begin{array}{l}5.63 \\
(5.44)\end{array}$ & $\begin{array}{c}6.42 \\
(6.30)\end{array}$ & 59.9 \\
\hline 2d: $\left(m-\mathrm{C}_{4} \mathrm{OPhO}\right)_{8} \mathrm{PcCu}$ & $\begin{array}{c}\mathrm{C}_{112} \mathrm{H}_{112} \mathrm{CuN}_{8} \mathrm{O}_{16} \\
(1889.68)\end{array}$ & 1887.75 & 1887.56 & $\begin{array}{l}71.09 \\
(71.19)\end{array}$ & $\begin{array}{l}5.93 \\
(5.97)\end{array}$ & $\begin{array}{l}6.18 \\
(5.93)\end{array}$ & 69.9 \\
\hline 2e: $\left(m-\mathrm{C}_{5} \mathrm{OPhO}\right)_{8} \mathrm{PcCu}$ & $\begin{array}{c}\mathrm{C}_{120} \mathrm{H}_{128} \mathrm{CuNN}_{8} \mathrm{O}_{16} \\
(2001.89)\end{array}$ & 1999.88 & 1999.63 & $\begin{array}{l}72.00 \\
(72.13)\end{array}$ & $\begin{array}{l}6.44 \\
(6.74)\end{array}$ & $\begin{array}{l}5.60 \\
(5.78)\end{array}$ & 47.4 \\
\hline 2f: $\left(m-\mathrm{C}_{6} \mathrm{OPhO}\right)_{8} \mathrm{PcCu}$ & $\begin{array}{c}\mathrm{C}_{128} \mathrm{H}_{144} \mathrm{CuN}_{8} \mathrm{O}_{16} \\
(2114.10)\end{array}$ & 2112.01 & 2112.07 & $\begin{array}{c}72.72 \\
(72.59)\end{array}$ & $\begin{array}{l}6.87 \\
(7.05)\end{array}$ & $\begin{array}{l}5.30 \\
(5.29)\end{array}$ & 55.9 \\
\hline 2g: $\left(m-\mathrm{C}_{7} \mathrm{OPhO}\right)_{8} \mathrm{PcCu}$ & $\begin{array}{c}\mathrm{C}_{136} \mathrm{H}_{160} \mathrm{CuN}_{8} \mathrm{O}_{16} \\
(2226.32)\end{array}$ & 2224.13 & 2223.95 & $\begin{array}{l}73.37 \\
(73.31)\end{array}$ & $\begin{array}{l}7.24 \\
(7.47)\end{array}$ & $\begin{array}{l}5.03 \\
(5.36)\end{array}$ & 58.7 \\
\hline 2h: $\left(m-\mathrm{C}_{8} \mathrm{OPhO}\right)_{8} \mathrm{PcCu}$ & $\begin{array}{c}\mathrm{C}_{144} \mathrm{H}_{176} \mathrm{CuN}_{8} \mathrm{O}_{16} \\
(2338.53)\end{array}$ & 2336.26 & 2336.36 & $\begin{array}{l}73.96 \\
(73.74)\end{array}$ & $\begin{array}{c}7.59 \\
(7.75)\end{array}$ & $\begin{array}{c}4.79 \\
(4.65)\end{array}$ & 44.7 \\
\hline 2i: $\left(m-\mathrm{C}_{9} \mathrm{OPhO}\right)_{8} \mathrm{PcCl}$ & $\begin{array}{c}\mathrm{C}_{152} \mathrm{H}_{192} \mathrm{CuN}_{8} \mathrm{O}_{16} \\
(2450.74)\end{array}$ & 2448.39 & 2448.27 & $\begin{array}{c}74.49 \\
(74.73)\end{array}$ & $\begin{array}{l}7.90 \\
(8.05)\end{array}$ & $\begin{array}{c}4.57 \\
(4.60)\end{array}$ & 43.1 \\
\hline
\end{tabular}

Table 2. UV-vis spectral data in chloroform of $\left(m-\mathrm{C}_{n} \mathrm{OPhO}\right)_{8} \mathrm{PcCu}$ $(n=3-9: 2 c-i)$.

\begin{tabular}{|c|c|c|c|c|c|c|c|}
\hline \multirow[b]{2}{*}{ Compound } & \multirow{2}{*}{\multicolumn{2}{|c|}{$\begin{array}{l}\text { Concentration } \\
\left(\times 10^{-6} \mathrm{~mol} / \mathrm{L}\right)\end{array}$}} & \multicolumn{4}{|c|}{$\lambda_{\max }(\mathrm{nm})(\log \varepsilon)$} & \multirow{2}{*}{$Q_{0-0}-$ band } \\
\hline & & & \multicolumn{2}{|l|}{ Soret-band } & \multicolumn{2}{|r|}{ Q-band } & \\
\hline $2 \mathbf{b}:\left(m-\mathrm{C}_{2} \mathrm{OPhO}\right)_{8} \mathrm{PcCu}^{\mathrm{a}}$ & & & & - & & & \\
\hline $2 \mathrm{c}:\left(m-\mathrm{C}_{3} \mathrm{OPhO}\right)_{8} \mathrm{PcCu}$ & 3.45 & $283.2(4.83)$ & $342.2(4.91)$ & $c a .383(4.54)^{\phi}$ & $613.8(4.63)$ & ca. $652(4.59)^{\circ}$ & o $682.4(5.33)$ \\
\hline $2 \mathrm{~d}:\left(\mathrm{m}-\mathrm{C}_{4} \mathrm{OPhO}\right)_{8} \mathrm{PcCu}$ & 3.45 & $282.5(4.84)$ & $340.4(4.92)$ & $c a .383(4.55)^{\phi}$ & $614.5(4.68)$ & ca.651(4.66) ${ }^{\circ}$ & $\circ 682.1(5.42)$ \\
\hline 2e: $\left(m-\mathrm{C}_{5} \mathrm{OPhO}\right)_{8} \mathrm{PcCu}$ & 3.46 & $283.7(4.82)$ & $343.9(4.90)$ & ca.382(4.53) & $614.9(4.65)$ & ca.651(4.63) $)^{\circ}$ & o $681.8(5.39)$ \\
\hline 2f: $\left(m-\mathrm{C}_{6} \mathrm{OPhO}\right)_{8} \mathrm{PcCu}$ & 3.46 & $283.9(4.87)$ & $341.0(4.94)$ & ca.382(4.57) $\phi^{\phi}$ & $613.5(4.69)$ & $\operatorname{ca.652(4.67)^{\circ }}$ & 。 $681.9(5.43)$ \\
\hline $2 \mathrm{~g}:\left(m-\mathrm{C}_{7} \mathrm{OPhO}\right)_{8} \mathrm{PcCu}$ & 3.45 & $283.8(4.87)$ & $339.5(4.95)$ & $c a .383(4.57)^{\phi}$ & $613.1(4.70)$ & ca. $654(4.66)^{\circ}$ & 。 $681.8(5.43)$ \\
\hline $2 \mathrm{~h}:\left(m-\mathrm{C}_{8} \mathrm{OPhO}\right)_{8} \mathrm{PcCu}$ & 3.46 & $283.7(4.88)$ & $342.1(4.94)$ & $c a .382(4.56)^{\phi}$ & $613.6(4.69)$ & $\operatorname{ca} .652(4.67)^{\circ}$ & $680.9(5.43)$ \\
\hline 2i: $\left(m-\mathrm{C}_{9} \mathrm{OPhO}\right)_{8} \mathrm{PcCu}$ & 3.44 & $283.8(4.90)$ & $342.6(4.97)$ & $c a .382(4.59)^{\phi}$ & $612.9(4.73)$ & ca.653(4.69) & 。 $681.6(5.46)$ \\
\hline
\end{tabular}




\section{2-2. Measurements}

Compounds 3b-i and $\mathbf{4 b}$-i synthesised here were identified by ${ }^{1} \mathrm{H}-\mathrm{NMR}$ measurements (BRUKER DRX-400) and FT-IR measurements (SHIMADZU FTIR-8400). The phthalocyanine 5 derivatives (2b-i) synthesised here were confirmed by MALDI-TOF mass spectra and elemental analyses (Table 1). MALDI- TOF mass spectra were measured using Autoflex III-2S. Elemental analyses were performed using a Perkin-Elmer elemental analyser 2400. Electronic absorption spectra were 10 measured using a Hitachi U-4100 automatic spectrophotometer (Table 2). The phase transition behaviour of $\mathbf{2} \mathbf{b}-\mathbf{i}$ was clarified by polarizing microscope observation (Nikon E-600 POL polarization microscope with hot stage consisting of Mettler FP-90 Central Processor) and differential scanning calorimetry 15 (Shimadzu DSC-50). The decomposition temperatures were measured with a Rigaku Thermo plus TG 8120 thermogravimeter. The mesophases were identified using a small angle X-ray diffractometer (Bruker Mac SAXS System) equipped with a hot stage (Mettler FP82HT hot stage, Mettler FP-90 Central 20 Processor). ${ }^{22}$ The measuring range of this small angle X-ray diffractometer is from $110 \AA$ to $3.0 \AA$, and the temperature is variable from rt. to $375^{\circ} \mathrm{C}$.

\section{RESULTS AND DISCUSSION}

\section{3-1. Synthesis}

${ }_{25}$ Table 1 summarizes the yields, elemental analysis and TOF-mass spectral results for the phthalocyanine derivatives $\mathbf{2 b - i}$ synthesised here. The electronic absorption spectra are summarized in Table 2. Figure 4 shows a spectrum of the representative derivative $\mathbf{2 h}$. However, UV-vis spectrum could 30 not be measured for $\mathbf{2} \mathbf{b}$ due to lack of the soluble solvent. Nevertheless, $\mathbf{2 b}$ could be judged from the elemental analysis and the TOF-mass spectrum (Table 1) that it was surely prepared.

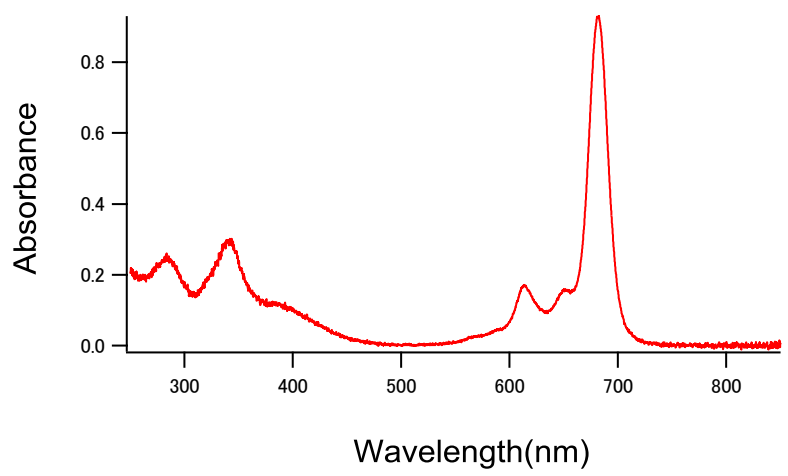

Fig. 4 UV-vis spectrum of $\left(m^{-} \mathrm{C}_{8} \mathrm{O}\right)_{8} \mathrm{PcCu}(2 \mathrm{~h})$ in chloroform 35

\section{3-2. Phase transition}

Table S1 summarizes phase transition behaviours of all the phthalocyanine derivatives $\left(m-\mathrm{C}_{n} \mathrm{OPhO}\right)_{8} \mathrm{PcCu}(n=1-20$ : 2a-o), which were established by using a polarizing optical microscopy 40 (POM), a differential scanning calorimeter (DSC) and a temperature-dependent small angle X-ray diffractometer.

The phase transition behaviours of the previously synthesized derivatives for $n=1,10-20(\mathbf{2 a}, \mathbf{2 j - 0})$ have already been reported. ${ }^{17,20,21}$ Therefore, Table 3 lists only the present ${ }_{45}$ derivatives for $\mathrm{n}=2-9(\mathbf{2} \mathbf{b}-\mathbf{i})$ and the representative previous derivatives for $n=1$ (2a) and $n=20(\mathbf{2 o})$. The phase transition behaviour of the present derivatives for $\mathbf{2} \mathbf{b}-\mathbf{i}$ are described below. $\left(m-\mathrm{C}_{2} \mathrm{OPhO}\right)_{8} \mathrm{PcCu}(\mathbf{2 b})$ having ethoxy groups $(n=2)$ shows a crystalline phase $\left(\mathrm{K}_{2}\right)$ at room temperature $(\mathrm{rt})$ for the 50 freshly prepared virgin sample. When it was heated, it transformed into another crystalline phase $\left(\mathrm{K}_{3}\right)$ at $84.2^{\circ} \mathrm{C}$ and then into $\mathrm{K}_{4}$ phase at $181.4^{\circ} \mathrm{C}$; on further heating, the $\mathrm{K}_{4}$ phase melted into a rectangular columnar $\left[\mathrm{Col}_{\mathrm{r}}(\mathrm{P} 2 \mathrm{~m})\right]$ mesophase at $274.7^{\circ} \mathrm{C}$ and it cleared into isotropic liquid (I.L.) at $354.1^{\circ} \mathrm{C}$. When the I.L. ${ }_{55}$ was cooled, it transformed into the $\mathrm{Col}_{\mathrm{r}}(\mathrm{P} 2 \mathrm{~m})$ mesophase and then another new crystalline phase $\left(\mathrm{K}_{1}\right)$, which was observed only for the non-virgin sample. It is very interesting that each of the short chain derivatives (2b-d) for $n=2-4$ shows only the $\mathrm{Col}_{\mathrm{r}}(\mathrm{P} 2 \mathrm{~m})$ mesophase, as can been seen from Table 3. The 60 propyloxy derivative (2e) for $n=5$ also shows the same $\mathrm{Col}_{\mathrm{r}}(\mathrm{P} 2 \mathrm{~m})$ mesophase and an additional monotropic hexagonal ordered columnar $\left(\mathrm{Col}_{\text {ho }}\right)$ mesophase. On the other hand, each of the moderately long chain derivatives (2f-i) for $n=6-9$ shows another type of rectangular columnar $\left[\mathrm{Col}_{\mathrm{ro}}\left(\mathrm{P} 2_{1} / \mathrm{a}\right)\right]$ mesophase 65 together with $\mathrm{Col}_{\text {ho }}$ mesophase(s).

It is very noteworthy that the derivative $\mathbf{2 h}$ for $n=8$ shows an unusual phase transition sequence. As can be seen from the DSC thermograms (Fig. S1) and the photomicrographs of $\mathbf{2 h}$ at various temperatures (Fig. S2), when the virgin sample of $\mathbf{2 h}$ was 70 heated from $\mathrm{rt}$, an unidentifiable $\mathrm{X}_{1}$ phase transformed into another unidentifiable $\mathrm{X}_{2}$ phase at $40.4^{\circ} \mathrm{C}$; the $\mathrm{X}_{2}$ phase transformed into $\mathrm{Col}_{\text {hol }}$ phase at $83.4^{\circ} \mathrm{C}$ and then into $\mathrm{Col}_{\mathrm{ro}}\left(\mathrm{P} 2_{1} / \mathrm{a}\right)$ phase at $118.9^{\circ} \mathrm{C}$. On further heating, it cleared into I.L. at $197.2^{\circ} \mathrm{C}$. Thus, the derivative $\mathbf{2 h}$ showed very unusual phase 75 transition sequence from a higher symmetry of $\mathrm{Col}_{\mathrm{h}}$ mesophase to a lower symmetry of $\mathrm{Col}_{\mathrm{r}}$ mesophase on heating stage. Usually, a lower symmetry of $\mathrm{Col}_{\mathrm{r}}$ mesophase transforms into a higher symmetry mesophase on heating stage. As can be seen also from Figs S1 and S2, when the I.L. heated over $205.0^{\circ} \mathrm{C}$ was 80 cooled down to $200.0^{\circ} \mathrm{C}$, it transformed into another hexagonal ordered columnar mesophase of $\mathrm{Col}_{\text {ho } 3}$; when the $\mathrm{Col}_{\text {ho3 }}$ at $200.0^{\circ} \mathrm{C}$ was rapidly cooled down to RT, a mixture of supercooled $\mathrm{Col}_{\mathrm{ho} 3}$ and partially resulted $\mathrm{Col}_{\mathrm{ho} 2}$. Interestingly, the lower symmetry of $\mathrm{Col}_{\mathrm{ro}}$ mesophase appeared only for the first 85 heating of the virgin sample during such usual observations by DSC and POM. Only when the supercooled $\mathrm{Col}_{\text {ho3 }}$ was held at $165.0^{\circ} \mathrm{C}$, the $\mathrm{Col}_{\mathrm{r}}$ (bright platelets) phase appeared with $\mathrm{Col}_{\mathrm{ho} 2}$ (dark hexagons), as can be seen from Fig S2.

In contrast with the moderately long chain derivatives $(\mathbf{2} \mathbf{f - i})$ 90 for $n=6-9$, each of the longer chain derivatives $\mathbf{2} \mathbf{j}-\mathbf{o}$ for $n=$ 10-20 shows only $\mathrm{Col}_{\mathrm{h}}$ mesophase(s), as can be seen from Table S1 and Table 3.

Thus, the mesomorphism of the present $\left(m-\mathrm{C}_{n} \mathrm{OPhO}\right)_{8} \mathrm{PcCu}$ derivatives (2a-o) for $n=1-20$ strongly depends on the alkoxy 95 chain length ( $n)$ : each of the short chain derivatives (2b-e) for $n=$ 2-5 shows the $\mathrm{Col}_{\mathrm{ro}}(\mathrm{P} 2 \mathrm{~m})$ mesophase; each of the moderately long chain derivatives (2f-i) for $n=6-9$ shows another type of rectangular columnar $\left[\mathrm{Col}_{\mathrm{ro}}\left(\mathrm{P} 2_{1} / \mathrm{a}\right)\right]$ mesophase together with $\mathrm{Col}_{\text {ho }}$ mesophase(s); each of the longer chain derivatives $\mathbf{2 j} \mathbf{j}-\mathbf{o}$ for $100 n=10-20$ shows $\mathrm{Col}_{\mathrm{h}}$ mesophase(s).

Table S2 summarizes small angle X-ray diffraction data of all 
the mesophases in the phthalocyanine derivatives $\mathbf{2 a - o}$. The data of $\left(m-\mathrm{C}_{n} \mathrm{OPhO}\right)_{8} \mathrm{PcCu}(n=1,10-20: \mathbf{2} \mathbf{a}, \mathbf{2} \mathbf{j}-\mathbf{o})$ reported previously $17,20,21$ are also included for the purpose of comparison.

Table 3. Phase transition temperatures and enthalpy changes of $\left(m-\mathrm{C}_{\mathrm{n}} \mathrm{OPhO}\right)_{8} \mathrm{PcCu}(\mathrm{n}=1,2-9$ and 20: $\mathbf{2 a}, \mathbf{2 b}-\mathbf{i}$ and $2 \mathbf{o})$.
As can be seen from this table, the phthalocyanine 5 derivatives

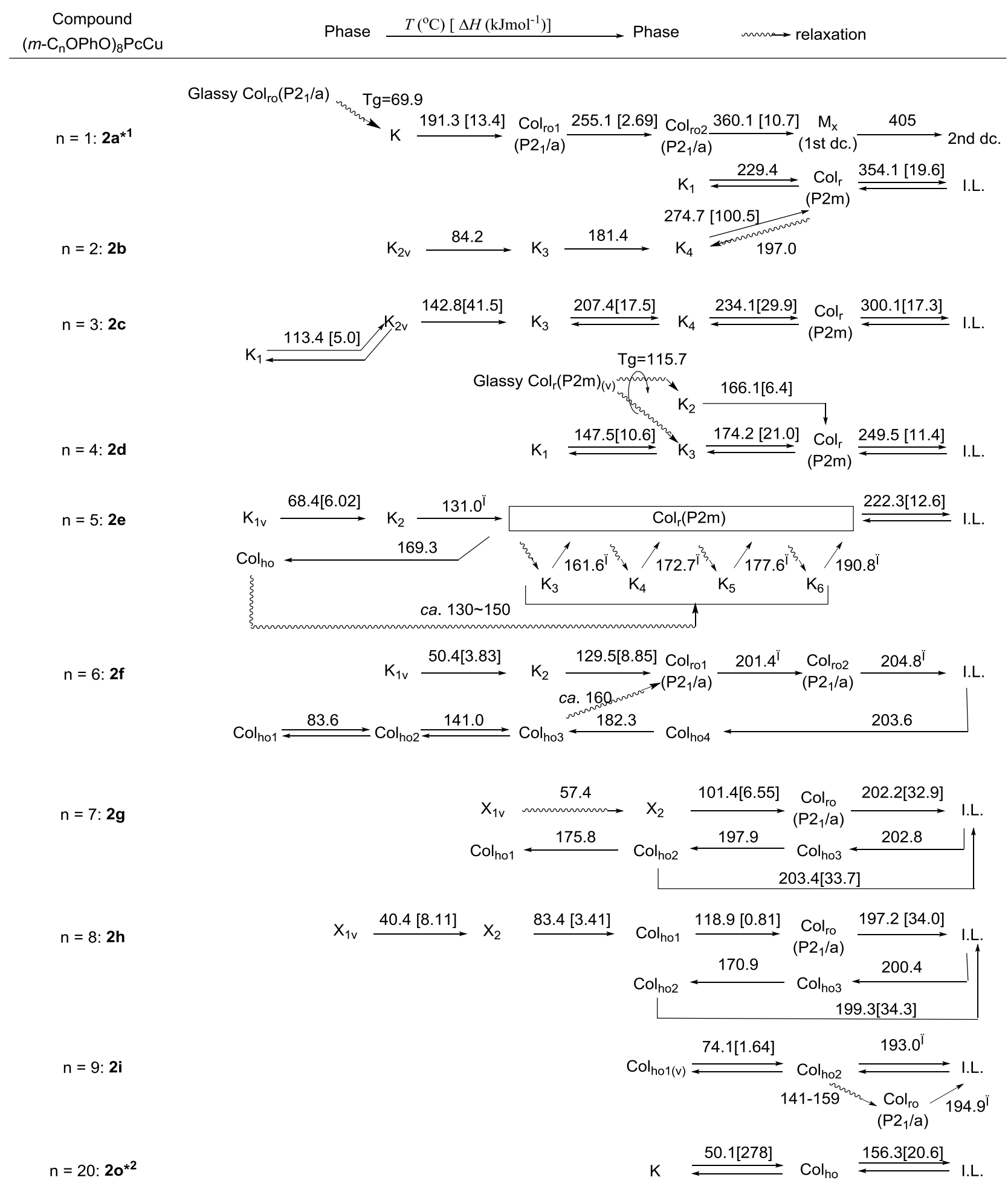

Phase nomenclature: $\mathrm{K}=\mathrm{crystal}, \mathrm{Col}_{\mathrm{ro}}=$ rectangular ordered columnar, $\mathrm{Col}_{\mathrm{ho}}=$ hexagonal ordered columnar mesophase, Col $\mathrm{hd}_{\mathrm{hd}}=$ hexagonal disordered columnar mesophase, I.L. = isotropic liquid and $v=$ virgin state. ${ }^{i}:$ These peaks were so close to calcurate the enthalpy changes. ${ }^{* 1}:$ Ref[17], ${ }^{* 2}:$ Ref[21]. 
2b-i $(n=2-9)$ synthesised in this study could be identified as columnar mesophases from the reflections due to two-dimensional lattices. Although each of the $\mathrm{Col}_{\text {ho4 }}$ phase of $\left(m-\mathrm{C}_{6} \mathrm{OPhO}\right)_{8} \mathrm{PcCu} \quad(\mathbf{2 f})$ and the $\mathrm{Col}_{\text {ho3 }}$ phase of $5\left(m-\mathrm{C}_{7} \mathrm{OPhO}\right)_{8} \mathrm{PcCu}(\mathbf{2 g})$ gave no (110) reflection from the 2D hexagonal lattice, they could be identified as a $\mathrm{Col}_{\text {ho }}$ phase from $\mathrm{Z}$ Value Calculation. ${ }^{23}$ When the reflection in the lowest angle is assigned as (1 000 ) of the two-dimensional hexagonal lattice, the $\mathrm{Z}$ value becomes 1 , which is consistent with the identification as $10 \mathrm{a} \mathrm{Col}_{\text {ho }}$ mesophase. This identification is also compatible with the textures mentioned below.
(A)

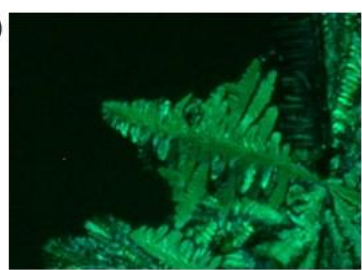

(B)

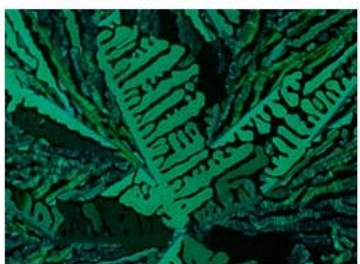

(C)

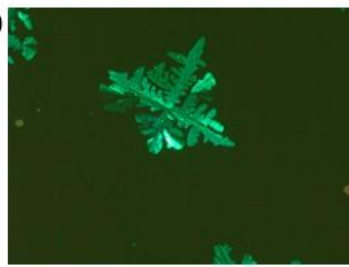

(D)

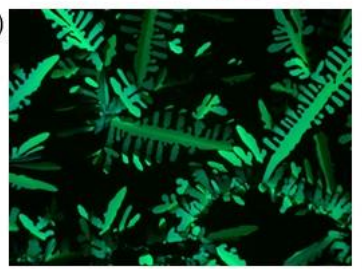

(E)

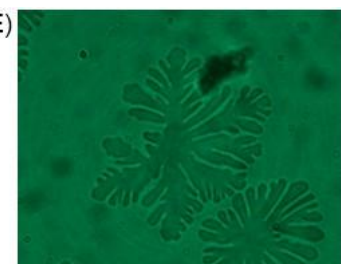

(F)

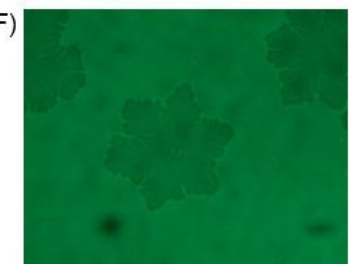

(G)

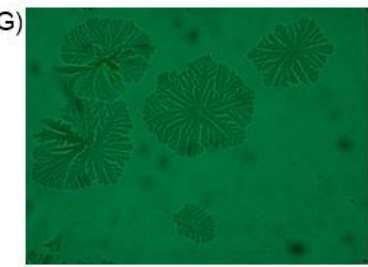

(H)

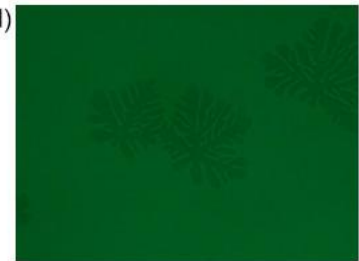

Fig. 5 Photomicrographs of $(\mathrm{A}) \mathrm{Col}_{\mathrm{r}}(\mathrm{P} 2 \mathrm{~m})$ of $\left(m^{-} \mathrm{C}_{2} \mathrm{OPhO}\right)_{8} \mathrm{PcCu}$ $(2 \mathrm{~b})$ at $340^{\circ} \mathrm{C}$; (B) $\mathrm{Col}_{\mathrm{r}}(\mathrm{P} 2 \mathrm{~m})$ of $\left(m-\mathrm{C}_{3} \mathrm{OPhO}\right)_{8} \mathrm{PcCu}(2 \mathrm{c})$ at $280^{\circ} \mathrm{C}$; (C) $\mathrm{Col}_{\mathrm{r}}(\mathrm{P} 2 \mathrm{~m})$ of $\left(\mathrm{m}^{-} \mathrm{C}_{4} \mathrm{OPhO}\right)_{8} \mathrm{PcCu}(2 \mathrm{~d})$ at $245^{\circ} \mathrm{C}$; (D)

$\mathrm{Col}_{\mathrm{r}}(\mathrm{P} 2 \mathrm{~m})$ of $\left(m-\mathrm{C}_{5} \mathrm{OPhO}\right)_{8} \mathrm{PcCu}(2 \mathrm{e})$ at $200^{\circ} \mathrm{C}$; (E) $\mathrm{Col}_{\text {ho } 4}$ of $\left(m^{-} \mathrm{C}_{6} \mathrm{OPhO}\right)_{8} \mathrm{PcCu}(2 \mathrm{f})$ at $200^{\circ} \mathrm{C}$; (F) $\mathrm{Col}_{\mathrm{ho3}}$ of $\left(m^{-} \mathrm{C}_{7} \mathrm{OPhO}\right)_{8} \mathrm{PcCu}$ $(2 \mathrm{~g})$ at $200^{\circ} \mathrm{C} ;(\mathrm{G}) \mathrm{Col}_{\text {ho3 }}$ of $\left(m^{-} \mathrm{C}_{8} \mathrm{OPhO}\right)_{8} \mathrm{PcCu}(2 \mathrm{~h})$ at $199.7^{\circ} \mathrm{C} ;(\mathrm{H})$ $\mathrm{Col}_{\text {ho2 }}$ of $\left(m^{-} \mathrm{C}_{9} \mathrm{OPhO}\right)_{8} \mathrm{PcCu}(2 \mathrm{i})$ at $196.5^{\circ} \mathrm{C}$.

20 Figure 5 shows the photomicrographs of the $\mathrm{Col}_{\mathrm{ro}}(\mathrm{P} 2 \mathrm{~m})$ phases of $\left(m-\mathrm{C}_{\mathrm{n}} \mathrm{OPhO}\right)_{8} \mathrm{PcCu}$ for $\mathrm{n}=2-5(\mathbf{2 b}-\mathbf{e})$ and the $\mathrm{Col}_{\mathrm{ho}}$ phases of $\left(m-\mathrm{C}_{\mathrm{n}} \mathrm{OPhO}\right)_{8} \mathrm{PcCu}$ for $\mathrm{n}=6-9(\mathbf{2 f - i})$. As can be seen from these photomicrographs, each of $\mathbf{2} \mathbf{b}$-e shows the dendritic textures characteristic to a $\mathrm{Col}_{\mathrm{r}}$ phase, and each of $\mathbf{2} \mathbf{f}-\mathbf{i}$ shows the 25 dendritic textures characteristic to a $\mathrm{Col}_{\mathrm{h}}$ phase having a $\mathrm{C}_{6}$ symmetry. Furthermore, they showed no birefringence resulted from homeotropic alignment. Thus, these $\mathrm{Col}_{\mathrm{ro}}(\mathrm{P} 2 \mathrm{~m})$ of $\mathbf{2 b}-\mathbf{e}$ and $\mathrm{Col}_{\text {ho }}$ phases of $\mathbf{2 f - i}$ could be identified from the XRD data and characteristic textures.

30 In Figure 6, the phase transition temperatures are plotted against the carbon number $(n)$ in the alkoxy chain. As can be seen from this figure, the derivative $\mathbf{2 a}(n=1)$ shows $\mathrm{Col}_{\mathrm{rol}}\left(\mathrm{P} 2_{1} / \mathrm{a}\right)$ and
$\mathrm{Col}_{\mathrm{ro} 2}\left(\mathrm{P} 2_{1} / \mathrm{a}\right)$ phases, and each of the derivatives $\mathbf{2 b}-\mathbf{d}(n=2-4)$ shows a $\mathrm{Col}_{\mathrm{r}}(\mathrm{P} 2 \mathrm{~m})$ phase. Therefore, the derivatives 2a-d $(n=$ 35 1-4) tend to show only the rectangular columnar $\left(\mathrm{Col}_{\mathrm{r}}\right)$ mesophase(s). On the other hand, the derivative $2 \mathbf{e}(n=5)$ shows an enantiotropic $\mathrm{Col}_{\mathrm{r}}(\mathrm{P} 2 \mathrm{~m})$ mesophase and a monotropic $\mathrm{Col}_{\mathrm{ho}}$ phase. Interestingly, the derivatives $\mathbf{2 f - h}(n=6-8)$ tend to show enantiotropic $\mathrm{Col}_{\text {ho }}$ phase(s) and $\mathrm{Col}_{\mathrm{ro}}\left(\mathrm{P} 2_{1} / \mathrm{a}\right)$ phase(s). As can be 40 seen from Table 3, the $\mathrm{Col}_{\mathrm{ro}}\left(\mathrm{P} 2_{1} / \mathrm{a}\right)$ phase(s) in the derivatives 2f-h $(n=6-8)$ appeared almost only for the freshly prepared virgin sample. Once it cleared into IL, the non-virgin sample gave only $\mathrm{Col}_{\text {ho }}$ mesophases. This phenomenon can be rationally explained by using Gibbs free energy vs. temperature (G-T) ${ }_{45}$ diagram. ${ }^{24}$ For a representative derivative $2 \mathbf{h}(n=8)$, the G-T diagram is illustrated in Figure S3. Although the derivatives $2 \mathbf{i}$ ( $n$ $=9$ ) also shows the $\mathrm{Col}_{\text {ho }}$ and $\mathrm{Col}_{\mathrm{ro}}\left(\mathrm{P} 2_{1} / \mathrm{a}\right)$ phase(s), the $\mathrm{Col}_{\mathrm{ro}}\left(\mathrm{P} 2_{1} / \mathrm{a}\right)$ phase was only observed for relaxation from the $\mathrm{Col}_{\text {ho }}$ phase. On the other hand, each of the derivatives $2 \mathbf{j}-\mathbf{o}(n=$ 50 10-20) shows only enantiotropic $\mathrm{Col}_{\mathrm{h}}$ phase(s), without any rectangular columnar $\left(\mathrm{Col}_{\mathrm{r}}\right)$ phases. Thus, the phase transition behaviour critically changes, between $n=1$ and 2 , between $n=5$ and 6 , and between $n=9$ and 10 , as indicated dotted lines in

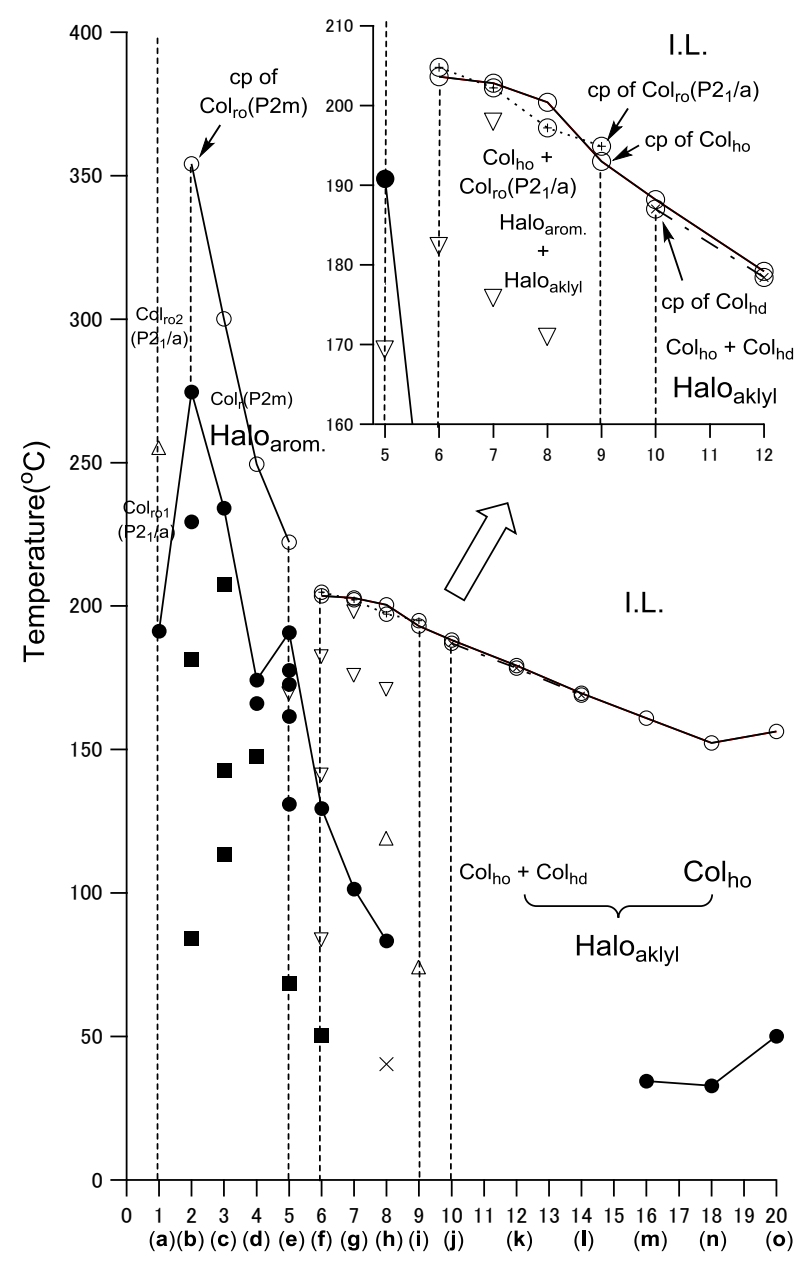

Number of carbon atoms in the alkoxy chain $(n)$

Fig. 6 Phase transition temperatures versus number of carbon atoms in the alkoxy chain $(\mathrm{n})$ for $\left(m^{-} \mathrm{C}_{\mathrm{n}} \mathrm{OPhO}\right)_{8} \mathrm{PcCu}(\mathrm{n}=1 \sim 20$ : 2a-o). 
Figure 6. For $\mathrm{n}=1$, the derivative decomposes at higher temperatures. Excluding this derivative, all the other derivatives can be classified into three groups: the short chain derivatives (2b-e) for $n=2-5$; the moderately long chain derivatives (2f-i) for $5 n=6-9$; the longer chain derivatives $2 \mathbf{j}-\mathbf{o}$ for $n=10-20$.

At these group boundaries between $n=5$ and 6 and between $\mathrm{n}=9$ and 10 , the driving force of mesomorphism may be changed. We predicted from this figure that the driving force of mesomorphism might be attributable to rotation or flip-flop of 10 only the bulky aromatic substituents for $n=2-5$, but melting of only the alkyl chains for $n=10-20$. In between for $n=6-9$, both of rotation of the bulky groups and melting of the longer alkyl groups may originate their mesomorphism. In order to confirm this hypothesis, we have carried out further detailed 15 temperature-dependent X-ray diffraction measurements.

\section{3-3. Temperature-dependent $X$-ray diffraction measurements}

For the detailed temperature-dependent X-ray diffraction measurements, we chose three representative derivatives from each of the three groups: $\mathbf{2 b}(n=2)$ for $n=2-5, \mathbf{2 h}(n=8)$ for $n=$ $206-9$, and $20(n=20)$ for $n=10-20$.

Figure 7 shows the X-ray diffraction patterns of the mesophases of $\mathbf{2 b}(n=2)$ at $290^{\circ} \mathrm{C}$ and $2 \mathbf{o}(n=20)$ at $100^{\circ} \mathrm{C}$. As can be seen from Figure 7(1), $\mathbf{2 b}(n=2)$ shows a halo at $2 \theta \cong$ $17^{\circ}$ (about $5.2 \AA$ ) (Halo arom: denoted as \#1) in the $\operatorname{Col}_{\mathrm{r}}(\mathrm{P} 2 \mathrm{~m})$ 25 mesophase, which is due to flip-flop of the bulky aromatic substituents. On the other hand, as can be seen from Figure 7(2), $20(n=20)$ shows a halo at $2 \theta \cong 18.5^{\circ}$ (about $\left.4.6 \AA\right)\left(\right.$ Halo $_{\text {alkyl }}$ : denoted as \#2) in the $\mathrm{Col}_{\text {ho }}$ mesophase, which is due to the molten alkyl chains. The Halo $\mathbf{H}_{\text {arom. }}$ of $\mathbf{2 b}(n=2)$ in Figure 7(1) could not 30 be observed in the X-ray diffraction pattern of $20(n=20)$ in Figure 7(2), whereas the Halo alkyl $_{\text {of }} \mathbf{2 0}(n=20)$ in Figure 7(2) could not be observed in the X-ray diffraction pattern of $\mathbf{2} \mathbf{b}(n=$ $2)$ in Figure 7(1). That means that the mesomorphism of $\mathbf{2} \mathbf{b}(n=$ 2 ) is induced only by the driving force originated from flip-flop 35 of the bulky substituents, but that the mesomorphism of $20(n=$ 20 ) is induced only by the driving force originated from the molten alkyl chains.

\section{(1) 2b $(n=2): \mathrm{Col}_{\mathrm{r}}(\mathrm{P} 2 \mathrm{~m})$ at $290^{\circ} \mathrm{C}$ (2) $20(n=20): \mathrm{Col}_{\mathrm{ho}}$ at $100^{\circ} \mathrm{C}$}

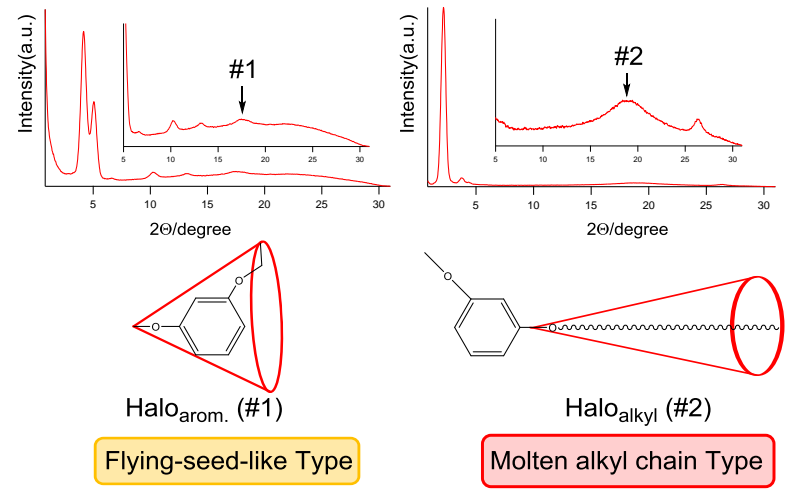

Fig. 7 Two different types of halos in the X-ray diffraction patterns of (1) $\left(m-\mathrm{C}_{2} \mathrm{OPhO}\right)_{8} \mathrm{PcCu}(2 \mathrm{~b})$ at $290^{\circ} \mathrm{C}$ and (2) $\left(m-\mathrm{C}_{20} \mathrm{OPhO}\right)_{8} \mathrm{PcCu}(20)$ at $100^{\circ} \mathrm{C}$.

In Figure 8 are plotted the spacing values of Halo arom. $_{\text {( }}$ 1) of $\mathbf{2} \mathbf{b}(n=2)$ and $\mathbf{H a l o}_{\text {alkyl }}(\# 2)$ of $\mathbf{2 0}(n=20)$ against temperature. As can be seen from this figure, both Halo $_{\text {arom. }}$ (\#1) and Halo alkyl $_{1}$
${ }_{45}$ (\#2) show their abrupt value jumps at the phase transition temperatures from crystal $(\mathrm{K})$ to columnar $(\mathrm{Col})$ mesophase. It is

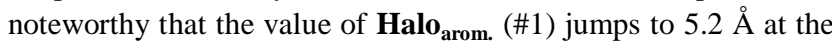
$\mathrm{K}-\mathrm{Col}$ phase transition temperature, $274.7^{\circ} \mathrm{C}$, whereas the Halo $_{\text {alkyl }}$ (\#2) jumps to $4.6 \AA$ at the $\mathrm{K}-\mathrm{Col}$ phase transition 50 temperature, $53.7^{\circ} \mathrm{C}$. Furthermore, the spacing value of Halo alkyl (\#2) gradually increases from $4.6 \AA$ to $4.8 \AA$ with elevating the temperature to $140^{\circ} \mathrm{C}$. Thus, the spacing values of $\mathbf{H a l o}_{\text {arom. }}(\# 1)$

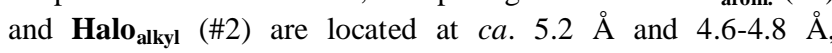
respectively. Therefore, we can distinguish Halo $_{\text {arom. }}$ (\#1) and ${ }_{55} \mathbf{H a l o}_{\text {alkyl }}$ (\#2) from their spacing values.

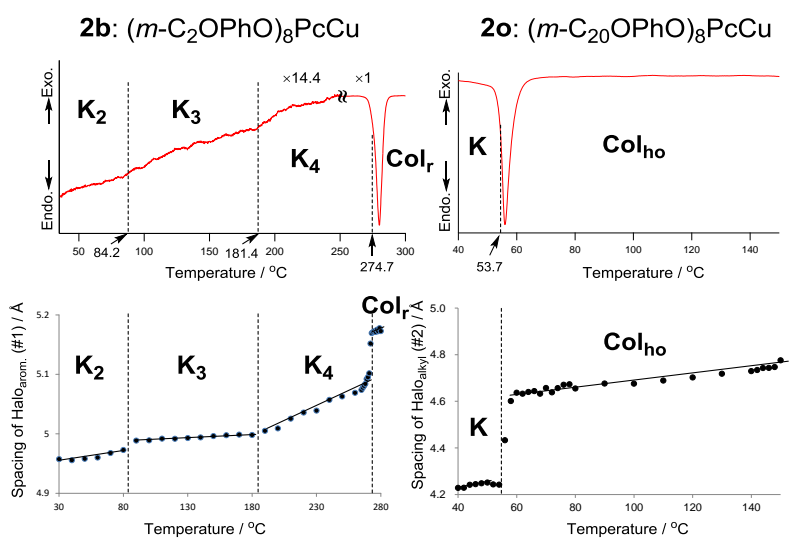

Fig. 8 Thermal dependence of the spacing of halos due to the flip-flopping aromatic groups (ca. $5.2 \AA)$ of $\left(m^{-} \mathrm{C}_{2} \mathrm{OPhO}\right)_{8} \mathrm{PcCu}$

(2b) and the molten alkyl groups (ca. 4.6 4.8 $\AA$ ) of $\left(m-\mathrm{C}_{20} \mathrm{OPhO}\right)_{8} \mathrm{PcCu}(2 \mathrm{o})$

Figure 9 shows the X-ray diffraction patterns for the moderately long chain derivative $2 \mathrm{~h}(\mathrm{n}=8)$ showing an unusual phase transition sequence from the $\mathrm{Col}_{\text {ho }}$ phase to the $\mathrm{Col}_{\mathrm{ro}}\left(\mathrm{P} 2_{1} / \mathrm{a}\right)$ phase on heating stage. This is completely reverse to the normal ${ }_{65}$ phase transition sequence. Usually, a phase transition occurs from a lower symmetry of $\mathrm{Col}_{\text {ro }}$ mesophase to a higher symmetry of $\mathrm{Col}_{\text {ho }}$ mesophase. This reverse sequence may be attributed to both flip-flop of the bulky aromatic substituents and melting of the

$\left(m-\mathrm{C}_{8} \mathrm{OPhO}\right)_{8} \mathrm{PcCu}(\mathbf{2 h})$
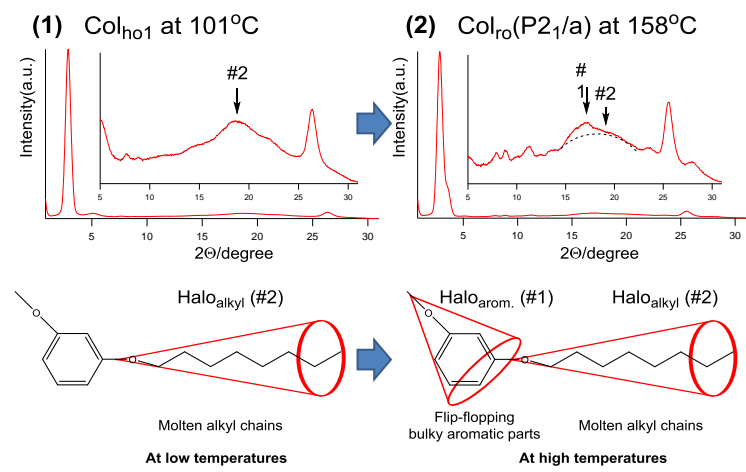

70 Fig. 9 X-ray diffraction patterns of $\left(\mathrm{m}^{-} \mathrm{C}_{8} \mathrm{OPhO}\right)_{8} \mathrm{PcCu}(\mathbf{2 h})$ at (1) $101^{\circ} \mathrm{C}$ and $(2) 158^{\circ} \mathrm{C}$.

alkyl chains. As can be seen from the X-ray diffraction patterns of $\mathrm{Col}_{\text {hol }}$ phase shown in Figure 9(1), Halo alkyl (\#2) only appears. On the other hand, as can been seen from the X-ray diffraction 75 patterns of $\mathrm{Col}_{\mathrm{ro}}\left(\mathrm{P} 2_{1} / \mathrm{a}\right)$ phase shown in Figure 9(2), Halo

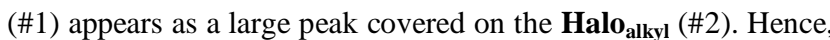
in the lower temperature region, the alkyl chain only melts to 
show the $\mathrm{Col}_{\text {hol }}$ phase, but in the higher temperature region, the bulky aromatic substituents also flip-flop to show the $\mathrm{Col}_{\mathrm{ro}}\left(\mathrm{P} 2_{1} / \mathrm{a}\right)$ phase.

To further confirm this phenomenon, we carried out fine $5 \mathrm{X}$-ray diffraction measurements by changing the temperature in small increments. Figure 10 shows the $\mathrm{X}$-ray diffraction patterns of $\left(m-\mathrm{C}_{8} \mathrm{OPhO}\right)_{8} \mathrm{PcCu}(\mathbf{2 h})$ measured in an angle region from 5 to 31 degree at 32 different temperatures. As can be seen from this figure, the halo due to the molten alkyl chains $\left(\right.$ Halo $_{\text {alkyl }}$ \#2) 10 appeared for $\mathrm{X}_{2}$ phase (blue lines) and $\mathrm{Col}_{\text {hol }}$ phases (green lines), whereas the halo due to the flip-flopping aromatic groups (Halo arom: \#1) appeared only for $\mathrm{Col}_{\mathrm{ro}}$ phase (red lines). Apparently, the halo suddenly shifted to lower angle at the phase transition from $\mathrm{Col}_{\mathrm{hol}}$ phase to $\mathrm{Col}_{\mathrm{ro}}$ phase.

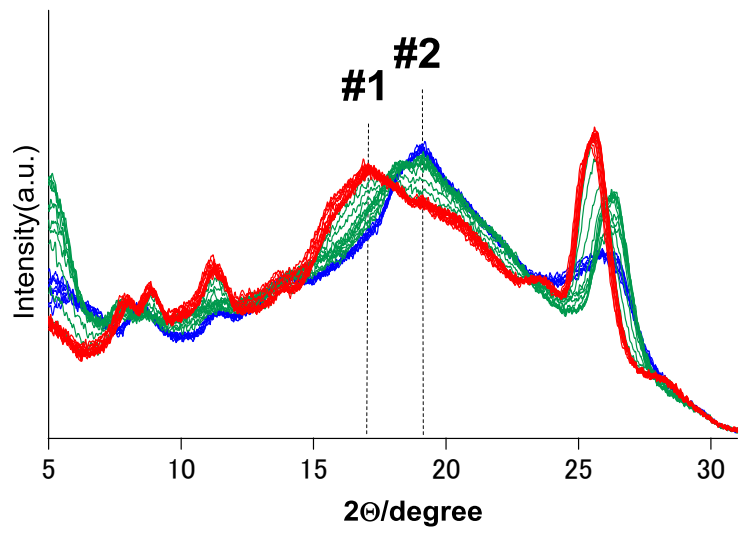

15 Fig. 10 Temperature-dependent $\mathrm{X}$-ray diffraction patterns of $\left(m-\mathrm{C}_{8} \mathrm{OPhO}\right)_{8} \mathrm{PcCu}(2 \mathrm{~h})$ in the rage of $2 \Theta=5 \sim 31$. Blue lines: $\mathrm{X}_{2}$ phase at $70,75,77,78,79,80$ and $81^{\circ} \mathrm{C}$. Green lines: Col $_{\text {ho } 1}$ phase at 87, 88, 90, 100, 105, 110,113,114, 115, 116, 117 and 118 ${ }^{\circ} \mathrm{C}$. Red lines: $\mathrm{Col}_{\text {ro }}$ phase at 119, 120, 121, 122, 123, 125, 130, 20 140, 150, 160, 170, 180 and $190{ }^{\circ} \mathrm{C}$. \#1: Haloarom. $=$ halo due to the flip-flopping aromatic groups (ca. $5.2 \AA$ ). \#2: Halo alkyl = halo due to the molten alkyl chains (ca. $4.6 \sim 4.8 \AA$ ).

In Figure 11, all the spacing values of Halo $_{\text {alkyl }}$ (\#2) and Halo $_{\text {arom. }}$ (\#1) of $\mathbf{2 h}$ are plotted against temperature on heating 25 stage. As can be seen from this figure, the spacing values of \#2 at about $4.8 \AA$ in the lower temperature region suddenly jumps to the spacing value of \#1 at about $5.2 \AA$ in the lower temperature region, just at the phase transition from the $\mathrm{Col}_{\text {hol }}$ mesophase to the $\mathrm{Col}_{\mathrm{ro}}\left(\mathrm{P} 2_{1} / \mathrm{a}\right)$ mesophase at $118.9^{\circ} \mathrm{C}$. Thus, it could be 30 confirmed that the $\mathrm{Col}_{\text {ho }}$ phase in the lower temperature region is originated by melting of the alkyl chains, and that the $\mathrm{Col}_{\mathrm{ro}}\left(\mathrm{P} 2_{1} / \mathrm{a}\right)$ phase in the higher temperature region is originated by flip-flop of the bulky aromatic substituents. Such a stepwise melting of these substituents may result in this present reverse phase 35 transition sequence from a higher symmetry of $\mathrm{Col}_{\mathrm{h}}$ mesophase to a lower symmetry of $\mathrm{Col}_{\mathrm{r}}$ mesophase for the moderately long chain derivative $\mathbf{2 h}(n=8)$. Furthermore, this mesogen is the first example switching from long alkyl chain type to flying-seed-like type in a discotic liquid crystal, so far as we know.

\section{${ }_{40}$ 4. CONCLUSION}

In this study, a series of phthalocyanine derivatives $\left(m-\mathrm{C}_{n} \mathrm{OPhO}\right)_{8} \mathrm{PcCu}(n=1-20: \mathbf{2 a - o})$ were prepared to investigate
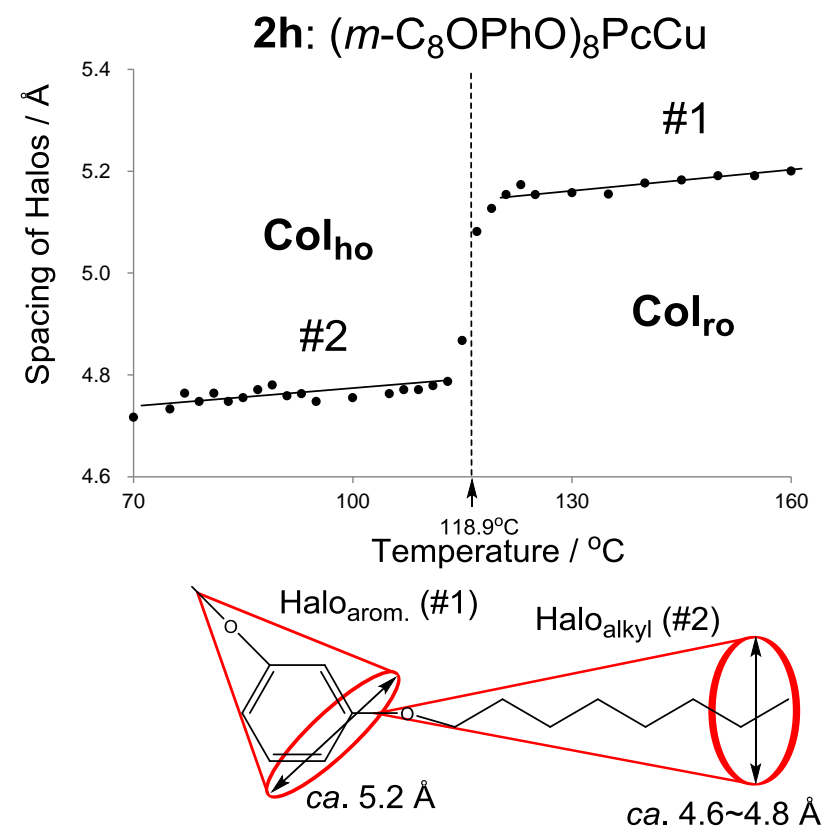

Fig. 11 Thermal dependence of the spacing of halos due to the molten alkyl groups (ca. 4.6 4.8 $\AA$ ) and the flip-flopping aromatic groups (ca. $5.2 \AA)$ of $\left(m^{-} \mathrm{C}_{8} \mathrm{OPhO}\right)_{8} \mathrm{PcCu}(2 \mathrm{~h})$.

the mesomorphism. The mesomorphism of the present $\left(m-\mathrm{C}_{n} \mathrm{OPhO}\right)_{8} \mathrm{PcCu}$ derivatives $(n=1-20: \mathbf{2 a - o})$ strongly depends on the alkoxy chain length $(n)$. The mesomorphism of the short 50 chain-substituted derivatives 2a-e for $n=1 \sim 5$ is flying-seed-like type induced by flip-flop of the peripheral bulky substituents, whereas the mesomorphism of the long chain-substituted derivatives $\mathbf{2} \mathbf{j}$-o for $\mathrm{n}=10 \sim 20$ is conventional molten alkyl chain type induced by melting of the long alkyl chains. The moderately

55 long chain derivatives (2f-i) for $n=6-9$ in between show both types of mesophases. The temperature-dependent small angle $\mathrm{X}$-ray diffraction studies revealed that a halo denoted as Halo $\mathbf{~ a r o m . ~}_{\text {. }}$ appeared in the short chain derivatives (2b-e) for $n=2-5$ at $\mathrm{d} \cong$ $5.2 \AA$ due to flip-flop of the bulky aromatic substituents, and that 60 another halo denoted as $\mathbf{H a l o}_{\text {alkyl }}$ appeared in the longer chain derivatives $2 \mathbf{j}$-o for $n=10-20$ at $\mathrm{d} \cong 4.6-4.8 \AA$ due to melting of the long alkyl chains. Therefore, we can distinguish the type of mesophase from Halo $\mathbf{H a m}_{\text {arom. }}$ and $\mathbf{H a l o}_{\text {alkyl }}$. Very interestingly, the moderately long chain derivative $\left(m-\mathrm{C}_{8} \mathrm{OPhO}\right)_{8} \mathrm{PcCu}(\mathbf{2 h})$ gave ${ }_{65}$ the $\mathbf{H a l o}_{\text {alkyl }}$ in the lower temperature mesophase of $\mathrm{Col}_{\text {ho }}$ at about $4.8 \AA$, and the Halo $_{\text {arom }}$ in the higher temperature mesophase of $\mathrm{Col}_{\mathrm{ro}}\left(\mathrm{P} 2_{1} / \mathrm{a}\right)$ phase at about 5.2 $\AA$. This means that melting of the alkyl chains induces the $\mathrm{Col}_{\text {hol }}$ phase in the lower temperature region, but that flip-flop of the bulky aromatic 70 substituents induces the $\mathrm{Col}_{\mathrm{ro}}\left(\mathrm{P} 2_{1} / \mathrm{a}\right)$ phase in the higher temperature region. Furthermore, this unusual reverse phase transition sequence from a higher symmetry of $\mathrm{Col}_{h}$ mesophase to a lower symmetry of $\mathrm{Col}_{\mathrm{r}}$ mesophase is attributable to the stepwise melting of the substituents. To our best knowledge, this 75 mesogen (2h) is the first example switching from molten alkyl chain type to flying-seed-like type in a discotic liquid crystal.

\section{Notes}

${ }^{a}$ Smart Material Science and Technology, Interdisciplinary Graduate School of Science and Technology, Shinshu University, 1-15-1 Tokida, 
Ueda, 386-8567, Japan. E-mail: ko52517@shinshu-u.ac.jp; Fax:

+81-268-21-5492; Tel: +81-268-21-5492

${ }^{b}$ Comprehensive Analysis Centre for Science, Saitama University, 255

Shimo-okubo, Sakura-ku, Saitama 338-8570, Japan. E-mail:

5 yasutake@apc.saitama-u.ac.jp; Tel. +81-48-858-3671

\section{References}

1 H. Keller, Mol. Cryst. Liq. Cryst. 1973, 21, 1-48; V. Vill, Condensed Matter News 1992, 1, 25-28.

2 Database of liquid crystalline compounds for personal computer,

10 LiqCryst Version 5, ed. Vill V., LCI Publisher and Fujitsu Kyushu Systems Limited, 2010.

3 D. Vorländer, Ber. Dtsch. Chem. Ges., 1911, 43, 3120-3125.

4 D. Demus, H. Sackmann, and K. Seibert, Wiss. Z. Univ. Halle, Math.-Nat. R. 1970, 19, 47-62.

155 P. Ferloni, M. Sanesi, P. L. Tonelli and P. Franzosini, Z. Naturforsch. 1978, 33a, 240-242.

6 M. Sanesi, P. Ferloni, G. Spinolo and P. L. Tonelli, Z. Naturforsch. 1978, 33a, 386-388.

7 R. van Deun, J. Ramaekers, P. Nockemann, K. van Hecke, L. van

20 Meervelt and K. Binnemans, Eur. J. Inorg. Chem., 2005, 563-571.

8 K. Ohta, T. Shibuya and M. Ando, J. Mater. Chem., 2006, 16, 3635-3639.

9 S. Basurto, S. Garcia, A. G. Neo, T. Torroba, C. F. Marcos, D. Miguel, J. Barbera, M. B. Ros and M. R. de la Fuente, Chem. Eur. J., $25 \quad 2005,11,5362-5376$.

10 M. Shimizu, M. Nata, K. Watanabe, T. Hiyama and S. Ujiie, Mol. Cryst. Liq. Cryst., 2005, 441, 237-241.

11 M. Shimizu, M. Nata, K. Mochida, T. Hiyama, S. Ujiie, M. Yoshio and T. Kato, Angew. Chem. Int. Ed., 2007, 46, 3055-3058.

3012 N. Usol'tseva, V. Bykova, G. Ananjeva and N. Zharnikova, Mol. Cryst. Liq. Cryst. 2004, 411, 1371-1378.

13 N. Zharnikova, N. Usol'tseva, E. Kudrik and M. Theakkat, J. Mater. Chem., 2009, 19, 3161-3167.

14 Y. Takagi, K. Ohta, S. Shimosugi, T. Fujii and E. Itoh, J. Mater. Chem., 2012, 22, 14418-14425.

15 A. Hachisuga, M. Yoshioka, K. Ohta and T. Itaya, J. Mater. Chem., 2013, 1, 5315-5321.

16 A. Ishikawa, K. Ohta and M. Yasutake, J. Porphyrins Phthalocyanines, 2015, 19, 639-650.

4017 M. Yoshioka, K. Ohta and M. Yasutake, RSC Advances, 2015, 5, 13828-13839.

18 A. Watarai, K. Ohta and M. Yasutake, J. Porphyrins Phthalocyanines, 2016, 20, 822-832.

19 K. Ohta, K. Adachi and M. Yasutake, J. Porphyrins Phthalocyanines, 2017; 21: 48-58.

20 M. Ichihara, A. Suzuki, K. Hatsusaka and K. Ohta, J. Porphyrins Phthalocyanines, 2007, 11, 503.

21 H. Sato, K. Igarashi, Y. Yama, M. Ichihara, E. Itoh and K. Ohta, J. Porphyrins Phthalocyanines, 2012, 16, 1148-1158.

5022 L. Tauchi, T. Nakagaki, M. Shimizu, E. Itoh, M. Yasutake and K. Ohta, J. Porphyrins Phthalocyanines, 2013, 17, 264-282.

23 K. Ohta, "Dimensionality and Hierarchy of Liquid Crystalline Phases: X-ray Structural Analysis of the Dimensional Assemblies", Shinshu University Institutional Repository, submitted on 11 May,

55 2013; http://hdl.handle.net/10091/17016; K. Ohta, "Identification of discotic mesophases by X-ray structure analysis," in "Introduction to Experiments in Liquid Crystal Science (Ekisho Kagaku Jikken Nyumon [in Japanese])," ed., Japanese Liquid Crystal Society, Chapter 2-(3), pp. 11-21, Sigma Shuppan, Tokyo, 2007; ISBN-13: 978-4915666490.

24 G-T diagram: e.g., see Fig. 1 in the following paper: H. Sato, Y. Sakagami, E. Itoh and K. Ohta, J. Porphyrins Phthalocyanines, 2012, 16, 1209-1216. This G-T diagram can rationally explain the mesophase appearance different between the virgin sample and the non-virgin sample. 